\title{
Management of plantar heel pain: a best practice guide synthesising systematic review with expert clinical reasoning and patient values
}

Dylan Morrissey ( $\nabla$ d.morrissey@qmul.ac.uk)

Queen Mary University London

Matthew Cotchett

La Trobe University https://orcid.org/0000-0001-6966-5324

Ahmed Said J'Bari

Queen Mary University London

Trevor Prior

4Homerton University NHS Foundation Trust, London

Bill Vicenzino

University of Queensland

lan Griffiths

Pure Sports Medicine

Michael Rathleff

Research Unit for General Practice, Aalborg

Halime Gulle

Queen Mary University London

Christian Barton

La Trobe University

\section{Systematic Review}

Keywords: Plantar fasciitis, pain, qualitative research, rehabilitation, education

Posted Date: June 22nd, 2020

DOI: https://doi.org/10.21203/rs.3.rs-36329/v1

License: (c) (i) This work is licensed under a Creative Commons Attribution 4.0 International License.

Read Full License 


\section{Abstract}

Objective To develop a Best Practice Guide for managing people with plantar heel pain (PwPHP)

Design a mixed methods design

Data sources Medline, Embase, CINAHL, SportsDiscus, Cochrane Central Register of Controlled Trials (CENTRAL), trial registries, reference lists, and citation tracking. Semi-structured interviews with world experts and a patient survey.

Eligibility criteria RCTs evaluating any intervention for PwPHP in any language were included subject to strict quality criteria. Trials with a sample size greater than 38 were considered for proof of efficacy. International experts were interviewed using a semi-structured approach and PwPHP surveyed online.

Results Forty PwPHP completed the survey and 14 experts were interviewed resulting in 7 themes and 38 sub-themes. Fifty-one eligible trials enrolled 4351 participants, with 10 interventions suitable to determine proof of efficacy. There was good agreement between the interview data and systematic review findings about taping (SMD: $-0.47,95 \% \mathrm{Cl}-0.88$ to -0.05 ) and stretching (SMD: $1.21,95 \% \mathrm{Cl} 0.78$ to 1.63 ) for first step pain in the short term, with clinical reasoning guidance to combine these interventions with education and footwear advice as a core approach. There was good agreement to stepped care with focussed shockwave for first step pain in the short term (OR: 1.72, 95\% Cl 1.14 to 2.61), medium term (SMD -1.31, 95\% Cl-2.01 to - 0.61) and long term (SMD -1.67, 95\% Cl-2.45 to -0.88) and radial shockwave for first step pain in the short term (OR: 1.66, 95\% $\mathrm{Cl} 1.00$ to 2.76 ) and long term (OR: 1.78, $95 \% \mathrm{Cl} 1.07$ to 2.96). Good agreement for stepped care was also revealed for custom foot orthoses for pain in the short term (SMD: $-0.41,95 \% \mathrm{Cl}-0.74$ to -0.07 ) and medium term (SMD: $-0.55,95 \% \mathrm{Cl}-1.02$ to $-0.09)$.

\section{Conclusion}

A best practice guide was formulated based on robust evidence, with application guided by expert reasoning and patients' perspectives.

Systematic review registration Prospero CRD42018102227

\section{Background}

Plantar heel pain (PHP) is one of the commonest reasons for rearfoot pain and leads to reduced function and disability, with a prevalence ranging from $3.6 \%$ to $7.0 \%$ in the community. ${ }^{1-3}$ PHP predominantly affects sedentary middle-aged and older adults, but is estimated to contribute $8.0 \%$ of all injuries related to running. ${ }^{4}$ The condition is characterised by first step pain and during weight-bearing tasks, particularly after periods of rest. ${ }^{5}$ PHP is also associated with impaired health related quality of life including social isolation, a poor perception of health status and reduced functional capabilities. ${ }^{6}$ 
Many interventions are used to treat pain and improve function in people with PHP. Currently, the literature is dominated by systematic reviews and meta-analyses that include low quality trials with small sample sizes, which may inflate effect sizes and contain incorrect inferences. ${ }^{7}$ When evidence is no longer sparse, as is now the case for PHP interventions, then it is no longer justifiable to rely on low-quality studies to guide decisions. Two previously published Clinical Practice Guides for PHP, based on variable quality evidence, do not recommend one treatment over another, ${ }^{5} 8$ while a recent network meta-analysis included low quality studies and limited the interventions considered to extracorporeal shockwave therapy, exercise, corticosteroid injections, NSAID injections, oral NSAIDS, and orthoses thus limiting validity and clinical applicability. ${ }^{9}$ There is therefore a clear need for a definitive guide to management.

Importantly, decisions about practice cannot be guided solely by clinical trial findings. Treatment decisions should also be guided by expert clinical reasoning alongside the views of people with a lived experience of the condition. ${ }^{10}$ The aim of this review was to synthesise findings from high quality level II randomised controlled trials with clinical reasoning from clinician-researchers and the patient voice, in order to develop a Best Practice Guide suitable for immediate use in the clinical management of PHP.

\section{Methods}

The systematic review element was registered on Prospero (CRD42018102227), with no deviations from protocol. Funding was from the Private Physiotherapy Education Fund, in the UK, who took no part in the delivery of the research. The review adhered to the guidelines for good reporting of a mixed methods study (Supplementary file 1).

\section{Search methods}

The final search was October 2019 of Ovid MEDLINE (1950 to date), Ovid EMBASE (from 1988 to date), CINAHL (1982 to date) and SPORTDiscus were searched from inception (Supplementary file 1). In addition, we also searched ClinicalTrials.gov (http://clinicaltrials.gov/), the WHO International Clinical Trials Registry Platform (http://apps.who.int/trialsearch/), and ControlledTrials.com (http://www.isrctn.com/) for ongoing or completed trials that met the eligibility criteria. Finally, references of included studies and citing articles were used to identify trials not located through the database search. No language restrictions were applied. 


\section{Criteria for selecting studies}

\section{Type of studies}

Randomised controlled trials (RCTs) evaluating the effectiveness of any intervention for PHP were included in any language from inception. All other designs including quasi-experimental studies, letters to the editor, opinion pieces, editorials and conference abstracts were excluded.

\section{Characteristics of participants}

A RCT was included if the participants were diagnosed with PHP with explicit reference to pain on the underside of the heel that was most noticeable upon weight-bearing after periods of rest but also worse following prolonged weight bearing. All participants were over the age of 16 and had experienced symptoms of any duration. RCTs were excluded if participants' PHP was related to fractures, tumours, or infections or confounded by other conditions that might be rheumatological, neural, vascular, or dermatological in origin.

\section{Types of interventions}

Any RCT that investigated the effectiveness or efficacy of an intervention compared to another intervention, placebo, sham or wait and see was included, provided there was a follow-up of at least two weeks.

\section{Outcome measures}

Studies that reported at least one outcome that evaluated patient-reported pain, first step pain or footrelated function were included. Where multiple measures of the same outcome were reported, the author's main pre-determined measure was accepted, or the lower level of effect was selected in order to avoid making recommendations based on Type 1 errors, or to be overly influenced by small effects - the rationale being that an intervention that results in a meaningful effect size for a given outcome should be positive irrespective of the measure used. 
Short term was defined as one week to three months, medium as greater than three and up to six months and long term as greater than six months. Where multiple follow-ups within a time window were reported, the outcome at the latest time point within short and medium term, and closest to one year for long term was selected

\section{Data collection and analysis}

\section{Selection of studies and assessment of quality}

Results of the database search were exported to EndNote X8 (Thomson Reuters, New York, USA) and duplicates removed. Titles were initially screened against the eligibility criteria. Relevant titles and abstracts were independently assessed by pairs of reviewers with disagreements resolved by $M C$ and DM. The PEDro scale ${ }^{11}$ an 11 point scale that evaluates internal validity and statistical interpretability with higher scores indicating greater quality. The PEDro database was screened to determine if a study had previously been reviewed and a quality score assigned. If a study had been graded, one reviewer independently rated the study, or two reviewers performed the rating with any disagreements from either method being resolved by a third reviewer. A study that scored $\geq 8 / 10$ on the PEDro scale was considered to be of high quality and retained, ${ }^{12}$ as a strong correlation exists between the PEDro scale and the Cochrane risk of bias tool, indicating evidence for strong convergence validity. ${ }^{13}$

The risk of bias was evaluated at study level for all RCTs that scored $\geq 8 / 10$ using six specific items of the PEDro scale, chosen based on a review of the Cochrane Collaboration tool for assessing risk of bias ${ }^{14}$ and those factors considered to influence internal validity and the size of the effect in RCTs. ${ }^{15}$ The items included random sequence generation (Item 2), allocation concealment (Item 3), baseline comparability (Item 4), blinding of outcome assessors (Item 7), adequate follow up (Item 8) and intention to treat analysis (item 9). Studies that scored less than 5 out of six were considered to have a high risk of bias and excluded from the review.

\section{Data extraction and management}

A data extraction form was used to record outcome data related to pain, first step pain and function, by pairs of authors, and checked by a senior author (MC). The mean and SD, median and interquartile range, or difference in outcomes from baseline was extracted for continuous data. In addition, dichotomous data were extracted. Data was extracted for each outcome at all time points and entered into RevMan (V.5.3; Copenhagen: The Nordic Centre, The Cochrane Collaboration, 2014). 


\section{Dealing with missing data}

For studies that did not report the number of participants at specific time points, or where participants were lost to follow up, the analyses were based on intention to treat data. Where data was missing, or not in a usable format, we attempted to contact the corresponding author of the study. If a request for data was not provided, we attempted to calculate SDs from standard errors, confidence intervals or $p$ values, according to the Cochrane Handbook for Systematic reviews of Interventions. ${ }^{14}$

For studies that reported median and range data, we estimated the mean and variance from the median, range, and the size of a sample. Where the sample size was $>25$, the median value was used to estimate the mean. To estimate the variance for samples $15<\mathrm{n} £ 70$ and $>70$, we used the formula range/ 4 and range/ 6 respectively to calculate the SD. ${ }^{16}$

\section{Evaluating effectiveness and the strength of the evidence}

An intervention was considered to be effective if adequately powered included trials demonstrated (i) primary proof of superiority compared to a no treatment control, sham or placebo or (ii) secondary proof of superiority compared to another treatment of proven efficacy or (iii) secondary proof of equivalence results to another treatment of proven efficacy, with the previously proven treatment yielding similar results to the initial RCT proving its effectiveness. If an intervention was not found to be superior when compared to sham or placebo, it was deemed to be ineffective and if it was compared to another unproven intervention, and no difference in effect noted then it was regarded as not being adequately tested. Where there were conflicting results between different studies for any outcomes at any time-point, efficacy was resolved by meta-analysis.

Adequate sample size was calculated using G*Power (Universitat Dusseldorf, http://www.gpower.hhu.de/en.html) at a power of $80 \%$, a minimum important difference of 19 on a VAS score for first step pain, ${ }^{17}$ a standard deviation of 28 and $5 \%$ alpha level defining a minimum sample size of 38 per group. This outcome measure was chosen as it is arguably the pathognomonic feature of PHP. In addition, 38 per group also represented the lowest calculated sample size for all our pre-specified outcome measures (i.e. overall pain and function) that we evaluated. If studies did not have an adequate sample size to be considered for primary or secondary proof of efficacy, they were included in metaanalysis where their results could be pooled with an adequately powered study. 
To evaluate the overall strength of the evidence for each intervention, we used the levels of evidence system designed by Van Tulder et al., ${ }^{18}$ which was adapted to reflect study inclusion being limited to high quality studies and applied for both positive and negative findings. Evidence was rated as:

- Strong evidence/Positive effect: meta-analysis revealed multiple high-quality trials demonstrated efficacy/ a positive effect in favour of the intervention

- Moderate evidence/Positive effect: analysis revealed one high quality trial demonstrated efficacy/a positive effect in favour of the intervention

- Limited evidence/Positive effect: analysis revealed one high quality trial, which did not meet the required sample size, demonstrated efficacy/ a positive effect in favour of the intervention

- Strong evidence/Neutral effect: meta-analysis revealed multiple high-quality trials demonstrated no efficacy/evidence of no effect

- Moderate evidence/Neutral effect: analysis revealed one high quality trial demonstrated no efficacy/evidence of no effect

- Limited evidence/Neutral effect: analysis revealed one high quality trial, which did not meet the required sample size, demonstrated no efficacy/ evidence of no effect

- Conflicting: Where there were conflicting between-study intervention results for any outcomes at any time-point, efficacy was resolved by meta-analysis

\section{Data synthesis}

For studies reporting continuous data, we calculated standardised mean differences (SMD), irrespective of whether the outcomes were similar or different but ensuring only outcomes of a similar construct were combined. Effect sizes were set as $0.20-0.49$ being small, $0.50-0.79$ as medium and 0.80 or above as large. ${ }^{19}$ Final scores at each follow up period were evaluated in preference to mean or median change from baseline values, unless only change scores were available. ${ }^{14}$ For studies reporting dichotomous data, to represent treatment success, odds ratios (ORs) were calculated. For studies that included three or more active treatment arms, and reported continuous outcomes, the active arms were combined and compared to the control group, to avoid a unit of analysis error, ${ }^{20}$ using accepted methods according to the Cochrane Handbook for Systematic reviews of Interventions. ${ }^{14}$

Comparable studies, reporting continuous data, were pooled using an inverse variance weighting method within a random effects model. ${ }^{21}$ For dichotomous outcome variables, the Mantel-Haenszel method was used to estimate an association between a treatment and outcome. The chi squared test and $P$ statistic 
were used to evaluate statistical heterogeneity. Finally, to evaluate the overall effect of an intervention of proven efficacy, individual studies that evaluated a specific intervention were combined to determine the size of within group changes.

\section{Semi-structured interviews with international experts}

\section{Participants}

International experts, defined as having a minimum of 5 years of experience in a given setting and specialty in which they regularly encountered significant numbers of patients with PHP and had active involvement in PHP research, were purposively recruited. ${ }^{22}$ Experts were identified by the authors through recommendations of researchers that publish in the field of PHP and snowball sampling. All experts were invited to participate via e-mail.

In total, 14 expert clinicians, from 5 countries, were recruited and interviewed, of which 7 were physiotherapists, 6 were podiatrists (including 2 podiatric surgeons) and 1 was a rheumatologist. Experts had a mean monthly exposure to 9 patients with PHP per month and had published an average of 51 publications. (Supplementary file 1).

\section{Interview process}

One of two interviewers, (AH and DM) conducted each interview online or face-to-face lasting between 30 and 90 minutes. An interview topic guide, piloted before use, was constructed based on a preliminary literature search, discussions within the research team and emergent concepts from interviews conducted (Supplementary file 1). Questions explored interviewees' background, clinical reasoning when managing PHP, perceptions of the evidence and gaps in the literature. An online graphic containing descriptors of possible interventions was presented at mid-interview as a stimulus to discussion. ${ }^{23}$ Interviews were transcribed verbatim. Data was collected until saturation, defined as the stage at which no new patterns or themes emerged. ${ }^{24}$ Verbal informed consent was obtained prior to each interview and ethics approval was granted by the Queen Mary University of London Ethics of Research Committee, UK.

\section{Analysis of interview data}


Interviews were recorded, transcribed and analysed concomitantly with data collection using the Framework approach. ${ }^{24}$ This entailed construction of an initial thematic framework from the topic guide following a process of data familiarisation. The themes and subthemes continuously evolved throughout the process of analysis by identifying emerging topics and mapping the ideas and beliefs of the recruited experts. The framework was applied to the data by coding each section of the text to subthemes and grouping them into themes, subthemes and supporting quotations as an initial descriptive analysis. This analysis was extended to identify typologies and tensions in the data to combine with systematic review data and yield the Best Practice Guide (BPG).

\section{Patient and public involvement - Survey of patients with PHP}

Interim results of the evidence synthesis (Figure 3), were presented to patients using an online survey tool (www.surveymonkey.co.uk) (Supplementary file 1). Open questions were asked, which explored a persons' experience of living with PHP, understanding the nature of the PHP, expectations of clinicians, strengths of PHP management and areas for improvement. Results were analysed with the Framework approach. $^{24}$

\section{Best practice guide formulation}

The BPG was constructed using established pragmatic approaches to integration by interpreting the systematic review findings through the lens of the clinical reasoning and evidence perspectives derived from the expert interviews to generate a core approach (Figure 2) ${ }^{25}$ The evolving BPG was then amended based on the patient survey results. Further, a quantitative check of the positive experienced or expected features of high-quality management was matched to the content of the core approach, which patients had not seen, using counts of agreement. The BPG was amended based on the patient survey results and presented as summary infographics and explanatory text in order to facilitate dissemination to both patients and professionals. This approach enabled consideration of multiple relevant perspectives, and included both qualitative and quantitative findings, as per recommended approaches that facilitate breadth and depth of understanding alongside corroboration from multiple sources. ${ }^{25}$ The qualitative quantitative balance was pre-determined so that inclusion of interventions was prioritised for systematic review findings, whereas ways of applying these interventions came from the expert interviews and patient survey.

\section{Results}




\section{Search results}

A total of 11,765 studies were identified through electronic databases and clinical trial registries (Figure 1). After removal of duplicates, 6839 titles were screened. 6477 studies were subsequently excluded leaving 362 studies to be assessed for full text retrieval and PEDro analysis. Following the quality analysis, 51 studies met the eligibility criteria and were available for analysis (Figure 1). In total, the review included 4351 participants.

INSERT Figure 1. Flow diagram for study selection process

The characteristics of the included studies, including the treatment arms, outcome measures, and participant characteristics are detailed in Supplementary file 1. Most studies had a small sample size (mean=81, range 30 to 252). The mean duration of symptoms was 13 months (range 0.8 to 68.3 months) and $49 \%$ were female participants. Pain was evaluated in all studies either by a visual analogue scale or numerical pain rating scale. Other outcome measures included the Foot Function Index (9 studies), Roles and Maudsley score (7 studies), plantar fascia thickness (6 studies), Foot Health Status Questionnaire (10 studies), The Manchester-Oxford Foot Questionnaire (2 studies), Lower Extremity Functional Scale (3 studies), Foot Ankle Ability Measure (2 studies), Maryland Foot Score (1 study), Global Rating of Change (1 study), EQ-5D (1 Study), SF-36 (1 study) and the Foot and Ankle Outcome Score (1 study). Finally, the mean length of follow up was 20 weeks (range 2 - 104 weeks). Ninety six percent of studies evaluated outcomes in the short term, but only $27 \%$ in the medium and $13 \%$ in the long term.

Of the included studies, the type of interventions evaluated included ESWT $(n=14)$, foot orthoses $(n=8)$, night splints $(n=1)$, footwear $(n=1)$, flip-flop sandals $(n=1)$, magnetised insoles $(n=1)$, local injections [corticosteroid $(n=11)$, botulinum toxin A injections $(n=1)$, polydeoxyribonucleotide $(n=2)$, hyaluronate injection $(n=1)$, ozone $(n=2)$, micronized $\operatorname{dHACM}(n=1)$, platelet rich plasma $(n=1)]$ manual therapy and exercise $(n=2)$, low level laser $(n=2)$, radiation therapy $(n=1)$, pulsed radiofrequency $(n=2)$, stretching $(n=1)$, trigger point dry needling $(n=1)$, taping and iontophoresis $(n=1)$, taping $(n=1)$, electrolysis $(n=1)$ and wheatgrass cream $(n=1)$. 


\section{Quality assessment}

The quality analysis results for studies that met the eligibility criteria are included in Supplementary file 1. The PEDro scores ranged from $8 / 10$ to $10 / 10$. Four studies scored 10/10. 'Blinding of all therapists who administered the therapy' was the criterion least met by the included studies $(n=7)$. In contrast, item 11 ('the study provides both point measures and measures of variability for at least one outcome') was the item most successfully completed $(n=51)$.

\section{Evidence of efficacy}

Table 1 provides a summary of the efficacy and strength of the evidence for interventions that were included for primary or secondary proof of efficacy. Supplementary file 1 includes the short, medium- and long-term results for each trial included in the analysis; a summary of those studies with evidence against efficacy and forest plots for the effectiveness of foot orthoses and ESWT.

Table 1: Efficacy and strength of evidence for interventions considered for primary and secondary proof of efficacya 


\begin{tabular}{|c|c|c|c|c|c|}
\hline Intervention & \multicolumn{2}{|c|}{ Outcome measure } & Short term ${ }^{b}$ & Medium term ${ }^{b}$ & Long Term ${ }^{b}$ \\
\hline \multicolumn{6}{|c|}{ Interventions with Primary proof of efficacy } \\
\hline \multirow[t]{4}{*}{ Custom orthoses } & \multirow[t]{2}{*}{ Pain } & $\begin{array}{l}\text { Between } \\
\text { group } \\
\text { efficacy }\end{array}$ & $\begin{array}{l}\text { Strong Positive } 26- \\
29 \\
-0.41(-0.74 \text {, } \\
-0.07)\end{array}$ & $\begin{array}{l}\text { Limited } \\
\text { Positive }^{28} \\
-0.55(-1.02 \\
-0.09)\end{array}$ & $\begin{array}{l}\text { Moderate } \\
\text { Neutral }^{26} \\
\\
-0.04(-0.45 \\
0.37)\end{array}$ \\
\hline & & $\begin{array}{l}\text { Within group } \\
\text { outcome }\end{array}$ & $\begin{array}{l}-1.24(-1.49 \\
-1.00)^{26-30} \mathrm{c}\end{array}$ & $\begin{array}{l}-1.65(-2.18 \\
-1.12)^{28} \mathrm{c}\end{array}$ & \\
\hline & \multirow[t]{2}{*}{$\begin{array}{l}\text { First } \\
\text { step } \\
\text { pain }\end{array}$} & $\begin{array}{l}\text { Between } \\
\text { group } \\
\text { efficacy }\end{array}$ & $\begin{array}{l}\operatorname{Limited~Neutra|}_{29}^{27} \\
-0.32(-0.91,0.26)\end{array}$ & & \\
\hline & & $\begin{array}{l}\text { Within group } \\
\text { outcome }\end{array}$ & & & \\
\hline & \multirow[t]{2}{*}{ Function } & $\begin{array}{l}\text { Between } \\
\text { group } \\
\text { efficacy }\end{array}$ & $\begin{array}{l}\text { Moderate } \\
\text { Neutral }^{26} 2829 \\
-0.21(-0.48,0.06)\end{array}$ & $\begin{array}{l}\text { Limited } \\
\text { Neutral }^{28} \\
-0.39(-0.85 \\
0.07) \\
\end{array}$ & $\begin{array}{l}\text { Moderate } \\
\text { Neutral }{ }^{26} \\
-0.12(-0.53 \text {, } \\
0.29) \\
\end{array}$ \\
\hline & & $\begin{array}{l}\text { Within group } \\
\text { outcome }\end{array}$ & & & \\
\hline \multirow[t]{6}{*}{$\begin{array}{l}\text { Prefabricated } \\
\text { orthoses }\end{array}$} & \multirow[t]{2}{*}{ Pain } & $\begin{array}{l}\text { Between } \\
\text { group } \\
\text { efficacy }\end{array}$ & $\begin{array}{l}\text { Moderate } \\
\text { Neutral }^{26} 29 \\
-0.25(-0.59,0.09)\end{array}$ & & $\begin{array}{l}\text { Moderate } \\
\text { Neutral }^{26} \\
\\
-0.08(-0.50 \\
0.33)\end{array}$ \\
\hline & & $\begin{array}{l}\text { Within group } \\
\text { outcome }\end{array}$ & & & \\
\hline & \multirow[t]{2}{*}{$\begin{array}{l}\text { First } \\
\text { step } \\
\text { pain }\end{array}$} & $\begin{array}{l}\text { Between } \\
\text { group } \\
\text { efficacy }\end{array}$ & & & \\
\hline & & $\begin{array}{l}\text { Within group } \\
\text { outcome }\end{array}$ & & & \\
\hline & \multirow[t]{2}{*}{ Function } & $\begin{array}{l}\text { Between } \\
\text { group } \\
\text { efficacy }\end{array}$ & $\begin{array}{l}\text { Moderate } \\
\text { Neutral }^{26} 29 \\
-0.06(-0.40,0.28)\end{array}$ & & $\begin{array}{l}\text { Moderate } \\
\text { Neutral }{ }^{26} \\
-0.08(-0.50 \\
0.33) \\
\end{array}$ \\
\hline & & $\begin{array}{l}\text { Within group } \\
\text { outcome }\end{array}$ & & & \\
\hline \multirow[t]{2}{*}{ Magnetised insoles } & \multirow[t]{2}{*}{ Pain } & $\begin{array}{l}\text { Between } \\
\text { group } \\
\text { efficacy }\end{array}$ & $\begin{array}{l}\text { Moderate } \\
\text { Neutral }^{31} \\
0.00(-0.39,0.39) \\
\end{array}$ & & \\
\hline & & $\begin{array}{l}\text { Within group } \\
\text { outcome }\end{array}$ & & & \\
\hline \multirow[t]{3}{*}{ Radial ESWT } & \multirow[t]{3}{*}{ Pain } & $\begin{array}{l}\text { Between } \\
\text { group } \\
\text { efficacy }\end{array}$ & $\begin{array}{l}\text { Strong Positive }{ }^{32} \\
33\end{array}$ & $\begin{array}{l}\text { Positive } \\
\text { limited }^{33}\end{array}$ & $\begin{array}{l}\text { Strong } \\
\text { positive } 3233\end{array}$ \\
\hline & & & $-1.64(-4.33,1.06)$ & $\begin{array}{l}-3.77(-4.72, \\
-2.82)\end{array}$ & $\begin{array}{l}-0.78(-1.72 \\
0.15)\end{array}$ \\
\hline & & $\begin{array}{l}\text { Within group } \\
\text { outcome }\end{array}$ & $-3.78(-6.17$ & $-5.81(-8.05$ & $-6.41(-7.83$ \\
\hline
\end{tabular}




\begin{tabular}{|c|c|c|c|c|c|}
\hline & & & $-1.38)^{32-35}$ & $-3.57)^{3334}$ & $-4.99)^{32} 33$ \\
\hline & $\begin{array}{l}\text { First } \\
\text { step } \\
\text { pain }\end{array}$ & $\begin{array}{l}\text { Between } \\
\text { group } \\
\text { efficacy }\end{array}$ & $\begin{array}{l}\text { Moderate } \\
\text { Neutral }{ }^{32,}, \\
\\
\text { OR: } 1.66(0.9975 \text {, } \\
2.76) \\
\end{array}$ & & $\begin{array}{l}\text { Moderate } \\
\text { Positive }^{32} \\
\text { OR: } 1.78(1.07 \text {, } \\
2.96) \\
\end{array}$ \\
\hline & & $\begin{array}{l}\text { Within group } \\
\text { outcome }\end{array}$ & $\begin{array}{l}-1.19(-1.63 \\
-0.76)^{36}\end{array}$ & $\begin{array}{l}-1.74(-2.21, \\
-1.26)^{36}\end{array}$ & $\begin{array}{l}-2.93(-3.51 \\
-2.34)^{36},\end{array}$ \\
\hline & Function & $\begin{array}{l}\text { Between } \\
\text { group } \\
\text { efficacy }\end{array}$ & $\begin{array}{l}\text { Moderate } \\
\text { Positive }^{32}\end{array}$ & $\begin{array}{l}\text { Positive } \\
\text { Limited }^{33}\end{array}$ & $\begin{array}{l}\text { Positive } \\
\text { Limited }^{33}\end{array}$ \\
\hline & & & $\begin{array}{l}-0.35(-0.60, \\
-0.10)\end{array}$ & $\begin{array}{l}-2.39(-3.12 \\
-1.65)\end{array}$ & $\begin{array}{l}-0.90(-1.49 \\
-0.32)\end{array}$ \\
\hline & & $\begin{array}{l}\text { Within group } \\
\text { outcome }\end{array}$ & $\begin{array}{l}-3.47(-4.37 \\
-2.57)^{33},\end{array}$ & $\begin{array}{l}-4.57(-5.65 \\
-3.48)^{33},\end{array}$ & $\begin{array}{l}-2.81(-3.61, \\
-2.02)^{33 \mathrm{~b}}\end{array}$ \\
\hline Focused ESWT & Pain & $\begin{array}{l}\text { Between } \\
\text { group } \\
\text { efficacy }\end{array}$ & $\begin{array}{l}\text { Strong Positive }{ }^{37} \\
\mathbf{3 8}\end{array}$ & $\begin{array}{l}\text { Moderate } \\
\text { Neutral }^{30}\end{array}$ & \\
\hline & & & $\begin{array}{l}-0.31(-0.53 \\
-0.09)\end{array}$ & $\begin{array}{l}0.18(-0.24, \\
0.60)\end{array}$ & \\
\hline & & $\begin{array}{l}\text { Within group } \\
\text { outcome }\end{array}$ & $\begin{array}{l}-1.33(-1.72, \\
-0.94)^{29,30,3539} \\
40, c\end{array}$ & $\begin{array}{l}-1.40(-1.86 \\
-0.94)^{38}\end{array}$ & \\
\hline & $\begin{array}{l}\text { First } \\
\text { step } \\
\text { pain }\end{array}$ & $\begin{array}{l}\text { Between } \\
\text { group } \\
\text { efficacy }\end{array}$ & $\begin{array}{l}\text { Strong Positive }{ }^{37-} \\
39\end{array}$ & $\begin{array}{l}\text { Limited } \\
\text { Positive }\end{array}$ & $\begin{array}{l}\text { Limited } \\
\text { Positive }^{41}\end{array}$ \\
\hline & & & $\begin{array}{l}\text { OR: } 1.72(1.14, \\
2.61)\end{array}$ & $\begin{array}{l}-1.31(-2.01, \\
-0.61)\end{array}$ & $\begin{array}{l}-1.67(-2.45, \\
-0.88)\end{array}$ \\
\hline & & $\begin{array}{l}\text { Within group } \\
\text { outcome }\end{array}$ & $\begin{array}{l}-2.11(-3.48 \\
-0.75)^{40} 4243\end{array}$ & $\begin{array}{l}-2.84(-3.73 \\
-1.94)^{41}\end{array}$ & $\begin{array}{l}-3.33(-3.87 \\
-2.78)^{41}\end{array}$ \\
\hline & Function & $\begin{array}{l}\text { Between } \\
\text { group } \\
\text { efficacy }\end{array}$ & $\begin{array}{l}\text { Moderate } \\
\text { Positive } \\
\\
-0.36(-0.61 \\
-0.10)\end{array}$ & & \\
\hline & & $\begin{array}{l}\text { Within group } \\
\text { outcome }\end{array}$ & $\begin{array}{l}-1.26(-1.53 \\
-0.99)^{29}\end{array}$ & & \\
\hline $\begin{array}{l}\text { Combined Radial and } \\
\text { Focused ESWT }\end{array}$ & Pain & $\begin{array}{l}\text { Between } \\
\text { group } \\
\text { efficacy }\end{array}$ & $\begin{array}{l}\text { Strong positive }{ }^{32} \\
333738\end{array}$ & $\begin{array}{l}\text { Strong } \\
\text { neutral }^{33} 38\end{array}$ & \\
\hline & & & $\begin{array}{l}-0.82(-1.48 \\
-0.16)\end{array}$ & $\begin{array}{l}-1.77(-5.64, \\
2.09)\end{array}$ & \\
\hline & & $\begin{array}{l}\text { Within group } \\
\text { outcome }\end{array}$ & $\begin{array}{l}-2.72(-4.05 \\
-1.39)^{33-3538}\end{array}$ & $\begin{array}{l}-4.33(-7.55 \\
-1.12)^{33} 3438\end{array}$ & \\
\hline & $\begin{array}{l}\text { First } \\
\text { step } \\
\text { pain }\end{array}$ & $\begin{array}{l}\text { Between } \\
\text { group } \\
\text { efficacy }\end{array}$ & $\begin{array}{l}\text { Strong positive } 32 \\
37-39\end{array}$ & & $\begin{array}{l}\text { OR 1.95(1.22, } \\
3.12)^{32} 41\end{array}$ \\
\hline
\end{tabular}




\begin{tabular}{|c|c|c|c|c|}
\hline & & & $\begin{array}{l}\text { OR } 1.70(1.23 \\
2.34)\end{array}$ & \\
\hline & & $\begin{array}{l}\text { Within group } \\
\text { outcome }\end{array}$ & $\begin{array}{l}-1.79(-2.66 \\
-0.92)^{364243}\end{array}$ & $\begin{array}{l}-3.14(-3.54 \\
-2.74)^{364143}\end{array}$ \\
\hline & Function & $\begin{array}{l}\text { Between } \\
\text { group } \\
\text { efficacy }\end{array}$ & $\begin{array}{l}\text { Strong positive } 33 \\
37 \\
-1.03(-2.42,0.36)\end{array}$ & \\
\hline & & $\begin{array}{l}\text { Within group } \\
\text { outcome }\end{array}$ & $\begin{array}{l}-2.32(-4.49, \\
-0.16)^{33} 37\end{array}$ & \\
\hline \begin{tabular}{|l|} 
Dry needling \\
\end{tabular} & Pain & $\begin{array}{l}\text { Between } \\
\text { group } \\
\text { efficacy }\end{array}$ & $\begin{array}{l}\text { Moderate } \\
\text { Neutral }^{44} \\
-0.33(-0.76,0.10)\end{array}$ & \\
\hline Within group outcome & & & & \\
\hline & $\begin{array}{l}\text { First } \\
\text { step }\end{array}$ & $\begin{array}{l}\text { Between } \\
\text { group }\end{array}$ & $\begin{array}{l}\text { Moderate } \\
\text { Neutral } 44\end{array}$ & \\
\hline & & enicacy & $-0.42(-0.85,0.02)$ & \\
\hline & & $\begin{array}{l}\text { Within group } \\
\text { outcome }\end{array}$ & & \\
\hline & Function & $\begin{array}{l}\text { Between } \\
\text { group } \\
\text { efficacy }\end{array}$ & $\begin{array}{l}\text { Moderate } \\
\text { Neutral }^{44} \\
0.11(-0.31,0.54)\end{array}$ & \\
\hline & & $\begin{array}{l}\text { Within group } \\
\text { outcome }\end{array}$ & & \\
\hline Wheatgrass & Pain & $\begin{array}{l}\text { Between } \\
\text { group } \\
\text { efficacy }\end{array}$ & $\begin{array}{l}\text { Moderate } \\
\text { Neutral }{ }^{\mathbf{4 5}, \mathrm{c}}\end{array}$ & \\
\hline & & $\begin{array}{l}\text { Within group } \\
\text { outcome }\end{array}$ & & \\
\hline & Function & $\begin{array}{l}\text { Between } \\
\text { group } \\
\text { efficacy }\end{array}$ & $\begin{array}{l}\text { Moderate } \\
\text { Neutral } \mathbf{4 5}, \mathrm{c}\end{array}$ & \\
\hline & & $\begin{array}{l}\text { Within group } \\
\text { outcome }\end{array}$ & & \\
\hline Calf stretching & $\begin{array}{l}\text { First } \\
\text { step } \\
\text { pain }\end{array}$ & $\begin{array}{l}\text { Between } \\
\text { group } \\
\text { efficacy }\end{array}$ & $\begin{array}{l}\text { Moderate } \\
\text { Neutral }^{46} \\
-0.39(-0.80,0.03)\end{array}$ & \\
\hline & & $\begin{array}{l}\text { Within group } \\
\text { outcome }\end{array}$ & & \\
\hline & Pain & $\begin{array}{l}\text { Between } \\
\text { group } \\
\text { efficacy }\end{array}$ & $\begin{array}{l}\text { Moderate } \\
\text { Neutral }^{46} \\
0.00(-0.40,0.41)\end{array}$ & \\
\hline
\end{tabular}




\begin{tabular}{|c|c|c|c|c|c|}
\hline & & $\begin{array}{l}\text { Within group } \\
\text { outcome }\end{array}$ & & & \\
\hline & \multirow[t]{2}{*}{ Function } & $\begin{array}{l}\text { Between } \\
\text { group } \\
\text { efficacy }\end{array}$ & $\begin{array}{l}\text { Moderate } \\
\text { Neutral }^{46} \\
-0.24(-0.65,0.17)\end{array}$ & & \\
\hline & & $\begin{array}{l}\text { Within group } \\
\text { outcome }\end{array}$ & & & \\
\hline \multirow[t]{6}{*}{ Low dye taping } & \multirow[t]{2}{*}{$\begin{array}{l}\text { First } \\
\text { step } \\
\text { pain }\end{array}$} & $\begin{array}{l}\text { Between } \\
\text { group } \\
\text { efficacy }\end{array}$ & $\begin{array}{l}\text { Moderate } \\
\text { Positive }^{47} \\
-0.47(-0.88 \\
-0.05) \\
\end{array}$ & & \\
\hline & & $\begin{array}{l}\text { Within group } \\
\text { outcome }\end{array}$ & $\begin{array}{l}-1.21(-1.66 \\
-0.77)^{47}\end{array}$ & & \\
\hline & \multirow[t]{2}{*}{ Pain } & $\begin{array}{l}\text { Between } \\
\text { group } \\
\text { efficacy }\end{array}$ & $\begin{array}{l}\text { Moderate } \\
\text { Neutral }^{47} \\
0.30(-0.11,0.71)\end{array}$ & & \\
\hline & & $\begin{array}{l}\text { Within group } \\
\text { outcome }\end{array}$ & & & \\
\hline & \multirow[t]{2}{*}{ Function } & $\begin{array}{l}\text { Between } \\
\text { group } \\
\text { efficacy }\end{array}$ & $\begin{array}{l}\text { Moderate } \\
\text { Neutral }^{47} \\
-0.05(-0.46,0.36)\end{array}$ & & \\
\hline & & $\begin{array}{l}\text { Within group } \\
\text { outcome }\end{array}$ & & & \\
\hline \multicolumn{6}{|c|}{ Interventions with Secondary proof of efficacy } \\
\hline \multirow[t]{2}{*}{$\begin{array}{l}\text { Plantar fascia } \\
\text { stretching }\end{array}$} & \multirow[t]{2}{*}{$\begin{array}{l}\text { First } \\
\text { step } \\
\text { pain }\end{array}$} & $\begin{array}{l}\text { Between } \\
\text { group } \\
\text { efficacy }\end{array}$ & $\begin{array}{l}\text { Moderate } \\
\text { Positive }^{36} \\
1.21(0.78,1.63)\end{array}$ & $\begin{array}{l}\text { Moderate } \\
\text { Positive }^{36} \\
\\
0.64(0.24 \\
1.04)\end{array}$ & $\begin{array}{l}\text { Moderate } \\
\text { Neutral }^{36} \\
-0.04(-0.43, \\
0.35)\end{array}$ \\
\hline & & $\begin{array}{l}\text { Within group } \\
\text { outcome }\end{array}$ & $\begin{array}{l}-2.81(-3.35 \\
-2.27)^{36}\end{array}$ & $\begin{array}{l}-3.25(-3.83, \\
-2.67)^{36}\end{array}$ & \\
\hline
\end{tabular}

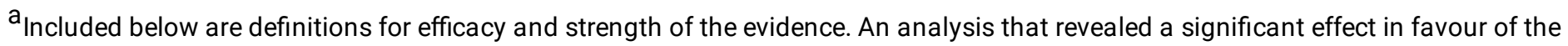
intervention was considered a positive effect. The strength of the evidence was rated as strong, moderate or limited based on the number of high quality trials and whether the trial was adequately powered:

Strong evidence/Positive effect: meta-analysis revealed multiple high-quality trials demonstrated efficacy/ a positive effect in favour of the intervention

Moderate evidence/Positive effect: analysis revealed one high quality trials demonstrated efficacy/ a positive effect in favour of the intervention

Limited evidence/Positive effect: analysis revealed one high quality trial, which did not meet the required sample size, demonstrated efficacy/ a positive effect in favour of the intervention

Strong evidence/Neutral effect: meta-analysis revealed multiple high-quality trials demonstrated no efficacy/evidence of no effect Moderate evidence/Neutral effect: analysis revealed one high quality trial demonstrated no efficacy/evidence of no effect Limited evidence/Neutral effect: analysis revealed one high quality trial, which did not meet the required sample size, demonstrated no efficacy/evidence of no effect 
${ }^{b}$ All effect sizes are reported as a Standardised Mean Difference $(95 \% \mathrm{Cl})$ unless otherwise stated.

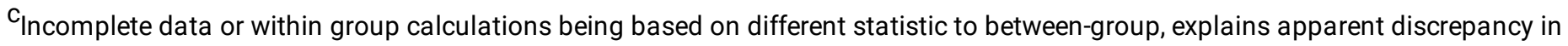
results and references used.

Of the 51 trials included in the review, nine interventions could be considered for primary proof of efficacy. The nine interventions included an evaluation of radial ESWT, ${ }^{32} 33$ focused ESWT, ${ }^{37-39} 41$ custom foot orthoses, ${ }^{26-29}$ prefabricated foot orthoses, ${ }^{26}$ dry needling, ${ }^{44}$ magnetised insoles, ${ }^{31}$ calf stretching, ${ }^{46}$ foot taping ${ }^{47}$ and wheatgrass cream. ${ }^{45}$ One trial that compared radial ESWT with plantar fascia stretching was considered for secondary proof of efficacy. ${ }^{36}$

Strong evidence was found for the efficacy of focused ESWT for overall pain (SMD: $-0.31,95 \% \mathrm{Cl}-0.53$ to -0.09 ), ${ }^{37} 38$ first step pain (OR: $1.7295 \% \mathrm{Cl} 1.14$ to 2.61$)^{37-39}$ in the short term and moderate evidence of effect for function in the short term (SMD: $-0.3695 \% \mathrm{Cl}-0.61$ to -0.10$).{ }^{37} \mathrm{No}$, or minimal side effects were reported in each study however the procedure was noted to be unpleasant for patients in both study reports and semi-structured interviews.

Significant and positive effects for pain in the short term were revealed for radial ESWT although pooling showed a large effect size but wide confidence intervals that crossed the line of no effect (SMD: -1.64 $95 \% \mathrm{Cl}-4.33$ to 1.06$){ }^{32}{ }^{33}$ Moderate evidence of efficacy was also revealed for first step pain in the long term (OR: $1.7895 \% \mathrm{Cl} 1.07$ to 2.96$).{ }^{32}$ Adverse events were reported as being minimal in each included study. Of note is that one high quality study found use of radial ESWT without local anaesthetic to be superior to ESWT with prior application of injected local anaesthetic. ${ }^{43}$ The systematic review findings were consistent with the opinions of experts who described the positive effect of ESWT:

"There's enough evidence to suggest that patients with heel pain that have shockwave therapy tend to have less pain on review than the patients that don't have shockwave therapy". (Expert 14) 
Strong evidence was found for the efficacy of custom foot orthoses versus sham for pain in the short term (SMD: $-0.4195 \% \mathrm{Cl}-0.74$ to -0.07$)$, although the results were conflicting. ${ }^{26-29}$ In addition, small effect sizes were reported for trials by Landorf, ${ }^{26}$ Oliveira ${ }^{28}$ and Wrobel, ${ }^{29}$ while a large effect was reported by Bishop. ${ }^{27}$ Qualitative data confirmed that foot orthoses, without specific reference to custom or prefabricated orthoses, can be used to unload tissues beneath the heel for short term relief particularly in situations where resting the foot is not feasible.

One study that met all the quality and power criteria evaluated the effectiveness of low dye taping and sham ultrasound versus sham ultrasound alone. ${ }^{47}$ There was moderate evidence of primary efficacy at 1 week for 'first-step' pain in favour of low dye taping (SMD: $-0.4795 \% \mathrm{Cl}-0.88$ to -0.05$)$. Some patients expressed the positive role for taping to alleviate symptoms:

"I think the strategies that I was given in the short term were helpful (e.g. taping and stretching" (Patient 12)

In addition, experts revealed that taping is a first line treatment that is an effective method to reduce pain in the short term and enhance patient confidence. Some experts used taping to predict the effectiveness of foot orthoses:

"If I tape them and their symptoms decrease and then I can say - okay, I think I can replicate what the tape is doing with either shoes or orthoses" (Expert 13)

There was moderate evidence of large effect that plantar fascia stretching is superior to radial ESWT for first step pain in the short term (SMD: $1.2195 \% \mathrm{Cl} 0.78$ to 1.63$)^{36}$ and of medium effect in the medium term (SMD: $0.6495 \% \mathrm{Cl} 0.24$ to 1.04 ) but not in the long term (SMD: $-0.0495 \% \mathrm{Cl}-0.43$ to 0.35 ). The sample was mainly early presenting PwPHP with acute PHP and this finding complements expert reasoning well, with there being clear guidance to continue stretching throughout rehabilitation, in a variety of ways targeting the plantar fascia and related structures: 
"They can feel an immediate response, and there seems to be some adaptation to this stretching, but again I would say this is definitely not the cure for this" (Expert 4)

\section{Expert interviews}

Interview transcript analysis revealed six themes and 30 subthemes. The first two themes concerned diagnosis and patient education (Table 2) and particularly influenced the core approach (Figure 2), along with the findings on stretching in the 'rehabilitation' theme alongside 'factors underlying management' and 'specific interventions' (Supplementary file 1- Expert Reasoning results) which had particular influence on the stepped approach to care (Figure 3). 'Perceptions of evidence' (Supplementary file 1) was the final theme and informed the recommendations made concerning application of specific interventions for patients recovering too slowly or not responding at all (Figure 3).

Table 2: Qualitative analysis of expert interview data pertaining to diagnosis and patient education 


\begin{tabular}{|c|c|c|}
\hline \multicolumn{3}{|c|}{ Findings } \\
\hline \multicolumn{3}{|c|}{ Theme 1: DIAGNOSIS } \\
\hline History & & \\
\hline $\begin{array}{l}\text { Overview of } \\
\text { key elements } \\
\text { to explore }\end{array}$ & $\begin{array}{l}\text { high repetitive use vs change of use; mechanical history essential } \\
\text { to establish; rest-activity balance important; typically insidious } \\
\text { onset but important to check injury; importance of ruling out other } \\
\text { causes; (inflammatory, tendinopathy and neuropathic } \\
\text { masqueraders); reduction with movement. }\end{array}$ & $\begin{array}{l}\text { Q: if you have had an } \\
\text { increase in weight, and } \\
\text { that's why you've got your } \\
\text { heel pain, then that's } \\
\text { probably a point of } \\
\text { discussion (11) } \\
\text { Q: was there an acute } \\
\text { incident, to rule out fat pad } \\
\text { contusion (10) } \\
\text { Q: those for whom it is part } \\
\text { of a systemic arthritis are } \\
\text { generally younger because } \\
\text { seronegative arthropathy is } \\
\text { often in a younger age } \\
\text { group (9) }\end{array}$ \\
\hline $\begin{array}{l}\text { relative } \\
\text { importance }\end{array}$ & $\begin{array}{l}\text { key factor in establishing diagnosis; sets priorities for physical and } \\
\text { imaging }\end{array}$ & $\begin{array}{l}\text { Q: the primary diagnosis, } \\
\text { when you first see someone, } \\
\text { is generally clinical (14) } \\
\text { Q: History essentially nails } \\
\text { the diagnosis (8) } \\
\text { Q: only time I would really } \\
\text { go for ultrasound would be } \\
\text { if I am suspecting a tear or a } \\
\text { rupture (8) }\end{array}$ \\
\hline $\begin{array}{l}\text { presentation } \\
\text { of pain }\end{array}$ & $\begin{array}{l}\text { am pain pathognomic; first step pain most informative; pain after } \\
\text { inactivity; well-localised to medial-inferior heel; worse at start and } \\
\text { at end / after aggravating activity; description as sharp at worst vs } \\
\text { ache at other times; mechanical vs psychosocial; }\end{array}$ & $\begin{array}{l}\text { Q: very localised pain at the } \\
\text { medial tubercle of the } \\
\text { calcaneum (3) } \\
\text { Q: first steps in the morning } \\
\ldots \text { after sitting for a long } \\
\text { time ... very good indication } \\
\text { (4) } \\
\text { Q: ...out of bed in the } \\
\text { morning it's like walking on } \\
\text { shattered glass or walking } \\
\text { on needles and pins (2) }\end{array}$ \\
\hline sub-groups & $\begin{array}{l}\text { lean vs high BMI; highly active vs relatively inactive; profession } \\
\text { may indicate risk; overweight and standing job a particular risk }\end{array}$ & $\begin{array}{l}\text { Q: one group is those with } \\
\text { high BMI, and they stand up } \\
\text { at work 7-8 hours a day, and } \\
\text { other group is the lean } \\
\text { runner maybe doing too } \\
\text { much too soon (2) } \\
\text { Q: you also have these } \\
\text { people standing a lot } \\
\text { standing } 8 \text { hours a day at } \\
\text { their working place (6) }\end{array}$ \\
\hline
\end{tabular}




\begin{tabular}{|c|c|c|}
\hline & & $\begin{array}{l}\text { Q: take a good history ... } \\
\text { profession and their sport } \\
\text { and fitness regime per week } \\
\text { (8) }\end{array}$ \\
\hline \multicolumn{3}{|l|}{ examination } \\
\hline $\begin{array}{l}\text { Physical } \\
\text { testing }\end{array}$ & $\begin{array}{l}\text { palpation at inferior medial heel (PF origin) or close to; check for } \\
\text { ruptures; look for compensation movements; calf flexibility a key } \\
\text { element }\end{array}$ & $\begin{array}{l}\text { Q: I could leave out the US } \\
\text { scan, but I would always do } \\
\text { a through history on the } \\
\text { patient, and palpate the } \\
\text { area (4) } \\
\text { Q: also check their calf } \\
\text { inflexibility (8) } \\
\text { Q: activate windlass } \\
\text { mechanism to see if plantar } \\
\text { fascia tightens (2) }\end{array}$ \\
\hline $\begin{array}{l}\text { Structures } \\
\text { of interest }\end{array}$ & $\begin{array}{l}\text { consider all aspects of fascia; consider old injuries (medial, lateral, } \\
\text { distal); tendinopathy, neuropathy and bone key differentials }\end{array}$ & $\begin{array}{l}\text { Q: squeeze the calcaneus ... } \\
\text { if that causes some } \\
\text { discomfort then I assume } \\
\text { that there's probably some } \\
\text { bony oedema (11) Q: } \\
\text { dorsiflex the hallux, } \\
\text { dorsiflex the ankle ... start } \\
\text { distally and palpate down } \\
\text { the plantar fascia and work } \\
\text { towards its origin (10) } \\
\text { Q: do some physical testing, } \\
\text { I rule out other } \\
\text { tendinopathy in the area (5) }\end{array}$ \\
\hline \multicolumn{3}{|l|}{ imaging } \\
\hline $\begin{array}{l}\text { decisions to } \\
\text { use imaging }\end{array}$ & $\begin{array}{l}\text { use is confirmatory not diagnostic; availability and speciality may } \\
\text { dictate use; subordinate to history and examination }\end{array}$ & $\begin{array}{l}\text { Q: US helps look at specific } \\
\text { portion of fascia; check for } \\
\text { tears and fibromas (8) } \\
\text { Q: if I do an US, diagnostic } \\
\text { US in someone, I cannot tell } \\
\text { them that they have PF, } \\
\text { that's how crazy it is (5) } \\
\text { Q: I think a lot of people go } \\
\text { wrong, they look at imaging } \\
\text { and try diagnosing, but } \\
\text { really it comes down to the } \\
\text { subjective features and the } \\
\text { clinical features (4) } \\
\text { Q: () }\end{array}$ \\
\hline $\begin{array}{l}\text { Perceptions } \\
\text { of utility }\end{array}$ & $\begin{array}{l}\text { sensitivity and specificity questionable; MRI unclear vs useful for } \\
\text { bone oedema; US useful to exclude tears and lumps; US }\end{array}$ & $\begin{array}{l}\text { Q: the more imaging work I } \\
\text { do the more I realise that }\end{array}$ \\
\hline
\end{tabular}


dimensions more useful than Doppler; changes likely bilateral even if unilateral pain there are other things that are going on (7)

Q: the other advantage is that MRI you can start to see there is inflammation, say, in the facets of the subtalar joint. You can start to see if there is some bone oedema (12)

Q: for the more resistant or long-term cases, then an MRI would be my investigation of choice (14)
Theme 2: Patient Education

Importance $\quad$ education key to prevent recurrence; importance as for all MSK
of Patient Education conditions; aetiology must be understood; key to patient engagement, self-management and treatment success; treatment rationale important for patient to learn; requires mixed communication methods; under-researched area; focus on key pain driver; relate to specific patient presentation; include physical and non-physical factors; reassure about positive long-term prognosis
Teaching about load Management
A primary goal of treatment; consider both static and dynamic weight-bearing load; change of overall load a risk factor for exacerbation; focus on function by unbundling erroneous patient perception of pain and pathology link; useful for patient to understand and self-manage a stepped approach to load increase with guidance; weight loss and associated metabolic factors poorly understood but impact on load management approach; need to address weight sensitively; therapists may not have weight management skills; key therapeutic effect mediator
Q: if we leave these maladaptive beliefs unchecked, then it will lead to chronicity (3)

Q: if they understand what the problem is and the course of it then it's easier to have compliance (6)

Q: if you don't address those issues then it could be that if you remove your orthotics, stop taping or stopped your stretching or whatever, the pain is just going to come back so that's where the education side of things is really important. (7)

Q: overarching thing is that you've got to individualise it for the person (11)

Q: load tolerance is probably a good way to describe the key treatment (3)

Q: obviously there's more load if you've got more weight, so if we can reduce that it's going to help reduce the load on the plantar fascia (1)

Q: get down to business and talk to him about his training programme and talk about how many miles they do a week (2) 


\begin{tabular}{|l|l|l|}
\hline $\begin{array}{l}\text { Advice on } \\
\text { footwear }\end{array}$ & $\begin{array}{l}\text { Comfort is key modification guide; consider softness, shock } \\
\text { absorption, rearfoot to forefoot drop and support; new shoes need } \\
\text { to be socially acceptable; can use to offload tissue }\end{array}$ & $\begin{array}{l}\text { Q: getting patients into } \\
\text { good footwear that has a } \\
\text { small heel on it, because it } \\
\text { takes the tension off the calf } \\
\text { muscle and therefore the } \\
\text { fascia, and having good } \\
\text { cushioning or shock } \\
\text { absorbency, are some key } \\
\text { factors (14) }\end{array}$ \\
& $\begin{array}{l}\text { Q: I don't think minimalist } \\
\text { (footwear) is made for } \\
\text { everybody (13) }\end{array}$ \\
\end{tabular}

INSERT Figure 2: Core approach to the management of plantar heel pain based on the best available evidence, expert opinion and the patient voice. The top layer ('DO') of taping, stretching and education are required initial interventions with each patient. The individual assessment ('DECIDE') is of which specific educational aspects are needed. LTCs $=$ long-term conditions. $\mathrm{RF}=$ rearfoot, $\mathrm{FF}=$ forefoot, $\mathrm{BMI}=$ body mass index".

INSERT Figure 3: Management approach for plantar heel pain when a person progressively fails to recover with addition of ESWT at 4 weeks if the core approach is not working and then addition of orthoses at 12 weeks if there is still sub-optimal improvement.

\section{Patient survey}

Forty people responded to the online survey with the Framework analysis resulting in one overarching theme of 'patient experience' with 8 sub-themes (Table 3). The quantitative check showed $95 \%$ of 266 specific treatment components or approach descriptions mentioned in the responses were consistent with the core approach initially derived from the review and expert interviews therefore indicating good agreement between the evidence, experts and patient experience.

\section{! 3: Framework analysis of 40 patient survey responses yielding 8 sub-themes.}




\begin{tabular}{|c|c|c|}
\hline Sub-Theme & $\begin{array}{c}\text { Findings } \\
\text { Illustrative quotes }\end{array}$ & \\
\hline $\begin{array}{l}\text { Thoughts on } \\
\text { condition } \\
\text { cause }\end{array}$ & $\begin{array}{l}\text { Foot arch height; age; activity pattern; new load increase; } \\
\text { long periods weight bearing; standing on hard surfaces; } \\
\text { minimally supportive footwear; limb length asymmetry; } \\
\text { rapidly changing load; altered gait; altered movement due } \\
\text { to other conditions }\end{array}$ & $\begin{array}{l}\text { Q: Walking on the outside } \\
\text { edge of my foot when I was } \\
\text { having pain in my second toe } \\
\text { (PN) } \\
\text { Q: Heel spurs, arthritis } \\
\text { Q: Long shifts on my feet in } \\
\text { facilities with hard floors. } \\
\text { Q: Excess loads with } \\
\text { inadequate progression } \\
\text { Q: a number of contributory } \\
\text { factors which is why is } \\
\text { occurred now }\end{array}$ \\
\hline $\begin{array}{l}\text { Thoughts on } \\
\text { pathology }\end{array}$ & $\begin{array}{l}\text { Tissue irritation; degeneration; inflammation; tearing; } \\
\text { inadequate tissue capacity; contracture }\end{array}$ & $\begin{array}{l}\text { Q: Tissue band has become } \\
\text { irritated through age/overuse } \\
\text { Q: It feels like it is tearing. I } \\
\text { think I have torn a ligament } \\
\text { Q: Inflamed damaged pf } \\
\text { which needs to heal/repair. } \\
\text { Q: Struggling to cope with the } \\
\text { demand and non adapted } \\
\text { tissue } \\
\text { Q: tendon contracture is } \\
\text { wanting to happen all the } \\
\text { time. }\end{array}$ \\
\hline Expectations & $\begin{array}{l}\text { More information; quick recovery-unrealised; exercise } \\
\text { programme esp. foot strengthening; pain elimination; } \\
\text { access to orthoses; specific treatments; better explanation } \\
\text { of treatment/condition and causes }\end{array}$ & $\begin{array}{l}\text { Q: Expected to get a steroid } \\
\text { shot and was hoping for deep } \\
\text { tissue manipulation to break } \\
\text { down the scaring or } \\
\text { thickening tissue. Wasn't } \\
\text { offered } \\
\text { Q: I assumed wrongly I would } \\
\text { need insoles. I expected to be } \\
\text { back on my feet within a few } \\
\text { weeks (very optimistic). } \\
\text { Q: as swift a recovery as } \\
\text { possible, relief from the pain } \\
\text { and programme of exercises } \\
\text { to treat }\end{array}$ \\
\hline $\begin{array}{l}\text { Needed } \\
\text { improvements }\end{array}$ & $\begin{array}{l}\text { Facilitation of earlier recognition by patients; better } \\
\text { communication as adherence promotion; } \\
\text { Intervention strategy for pain; Easier access to, and more } \\
\text { information on, specific treatments; Standardised } \\
\text { treatment across sectors; Clarity of treatment and } \\
\text { expectations; reduced waiting times }\end{array}$ & $\begin{array}{l}\text { Q: better understanding of } \\
\text { symptoms and types of } \\
\text { patients prone to PHP } \\
\text { Q: More explanation for the } \\
\text { mechanism of the symptoms }\end{array}$ \\
\hline
\end{tabular}

Page 23/39 


\begin{tabular}{|c|c|c|}
\hline & & $\begin{array}{l}\text { in order to motivate me to do } \\
\text { the exercise } \\
\text { Q: Get rid of the pain forever } \\
\text { Q: Standardised treatment } \\
\text { from NHS across the country. } \\
\text { I've gone private as Dr can't } \\
\text { refer. }\end{array}$ \\
\hline $\begin{array}{l}\text { Strengths of } \\
\text { management }\end{array}$ & $\begin{array}{l}\text { From no strengths to positive experiences; fast decisions; } \\
\text { specific interventions; clear plan; individual preferences } \\
\text { accounted for; detailed explanation; specific interventions }\end{array}$ & $\begin{array}{l}\text { Q: Range of options } \\
\text { considered and clearly } \\
\text { explained } \\
\text { Q: Spent time explaining in } \\
\text { detail the } \\
\text { condition/cause/treatment }\end{array}$ \\
\hline Experience & $\begin{array}{l}\text { Restricted activity; intermittent severe pain; reduced } \\
\text { exercise; altered activity; morning pain; painful; } \\
\text { emotionally affected; large impact on ADL; long, uncertain } \\
\text { recovery }\end{array}$ & in $\begin{array}{l}\text { Q: It restricted the activities I } \\
\text { wished to carry out } \\
\text { Q: It's very painful under my } \\
\text { heel when I get up in the } \\
\text { morning } \\
\text { Q: Miserable } 6 \text { months. Had a } \\
\text { huge impact on daily } \\
\text { activities. } \\
\text { Q: Very long process and } \\
\text { uncertain outcome }\end{array}$ \\
\hline $\begin{array}{l}\text { Key } \\
\text { information }\end{array}$ & $\begin{array}{l}\text { Time course of recovery; self-management advice; how } \\
\text { pain relief works; long term effects; explanation of what } \\
\text { was not done; unsure; statistics on usual timescales for } \\
\text { effects }\end{array}$ & $\begin{array}{l}\text { Q: What can I do to reduce my } \\
\text { pain and improve function } \\
\text { Q: Will pain reliever actually } \\
\text { address the issue or just } \\
\text { mask the pain? } \\
\text { Q: When they could make the } \\
\text { pain go away } \\
\text { Q: Expected outcome at the } \\
\text { end of rehab }\end{array}$ \\
\hline $\begin{array}{l}\text { Sources of } \\
\text { information }\end{array}$ & $\begin{array}{l}\text { Range of online methods predominated; clinicians, } \\
\text { friends, magazines; lack of clear guidance }\end{array}$ & $\begin{array}{l}\text { Q: I can google it all day, and there isn't } \\
\text { much out there } \\
\text { Q: Patient groups on Facebook aren't } \\
\text { even very helpful, because everyone } \\
\text { using them hasn't found relief. } \\
\text { Q: online forums, confusing as } \\
\text { everyone's cause is different } \\
\text { therefore treatment different }\end{array}$ \\
\hline
\end{tabular}




\section{Best practice guide}

The BPG was produced from synthesis of quantitative (review) and qualitative (expert interviews and patient survey) data. A core approach was determined (Figure 2) prior to stepped care for patients progressing slowly, or inadequately (Figure 3). The core approach consists of the evidence-based interventions of plantar fascia stretching and low-dye taping complemented by an individualised education approach, with all components recommended to be used for approximately 6 weeks before consideration of adjunctive interventions such as ESWT or orthoses, with the expert interviews strongly emphasising the need to implement an education and self-management approach prior to applying the interventions with the strongest evidence (Table 2).

"The nature of the condition is that you need to be doing a range of things, but all together for a sustained period of time" (Expert 14)

Of the three components of the core approach, taping and plantar fascia stretching should be universally applied and were annotated as 'DO' in Figure 2. Individualised decisions about education content are needed and were annotated as 'DECIDE' in Figure 2. Education content had four sub-areas. For load management, the keys were to reduce overall tissue compressive load by breaking up long periods of static load such as standing and reducing injurious compressive and stretch-related dynamic load from activities such as running in the more active population. For pain education, clarity about the meaning of pain and its relation to tissue state needs to be clearly understood, alongside realistic expectations of the prognosis being good but resolution likely to be slow. Techniques such as pain-monitoring were strongly recommended. The possible impact of other presenting long-term conditions and an adverse body mass need to be addressed. Finally, the requirement for footwear to be supportive, comfortable, incorporate a rearfoot to forefoot drop and be socially acceptable is required with specific advice to avoid barefoot walking and open sandals until symptoms have entirely resolved.

\footnotetext{
"the number one thing is educating (sic) PWPHP to have some understanding about the most likely reason they felt the pain, and then based on that, the key things that they need to do long term" (Expert 9)
} 
Where the core approach is only partially successful or taking greater than six weeks to yield suitable effects, adjunct interventions are recommended based on the strength of the quantitative evidence and expert reasoning. The use of simple but validated patient reported outcome measures, such as a global rating of change scale (Kamper et al., 2009) or equivalent may help guide these decisions. Patient experience had little influence on this section, with adjunctive interventions such as ESWT and orthoses having less prominence in responses. The primary recommendation was that ESWT - applied using either radial or focussed approaches - should be applied if a PwPHP is not deriving optimal benefit from the core approach as it has the strongest overall evidence.

Where the core approach and ESWT are still not successful, the stepped care approach recommended custom orthoses and if still not successful - as marked by an X in Figure 3 - then experimental approaches may be tried, although expert reasoning suggests that a return to the core approach and repeat application ensuring that accuracy and adherence is also a feasible fourth line approach. Where interventions have been tried and shown to be ineffective, they should not be used except in formal trials, whereas inadequately tested interventions with no primary or secondary proof of efficacy - such as injection therapy - where the evidence is inadequate or not present may be considered - again preferably in an RCT or with structured evaluation.

\section{Discussion}

A BPG has been produced with a pragmatic mixed-methods integration of systematic review of high quality RCTs, elicited expert clinical reasoning and a survey of patient values which therefore meets the majority of the relevant Agree II criteria, ${ }^{48}$ and the high quality criteria previously reported for guideline development. ${ }^{49}$ The BPG defines a core approach (Figure 2) to management which consists of the simple interventions of stretching ${ }^{36}$ and taping (labelled 'DO' in the figure) to support the plantar fascia, ${ }^{47}$ alongside less well-defined educational interventions (labelled 'DECIDE' in the figure). The interviews gave clear direction that this education should be based on individual assessment and include footwear advice to ensure comfort and social acceptability in shoes that allow a small rear-foot to forefoot drop; load management to break up long periods of static load or overly fast training changes in the more athletic populations; and advice on prompts to address co-morbidities such as type 2 diabetes (Supplementary file 1). Further, teaching patients the parameters required to self-monitor the pain response to activity and how to interpret pain with respect to tissue damage in order to allay fears of long-term consequences should help guide education packages. Communicating a realistic but positive prognosis came through strongly in the expert interviews and patients' responses. The patient survey showed very strong 
agreement with the content of the core intervention. Importantly, patient survey responses were not biased by knowledge of what the core intervention entailed, with the stimulus having been Figure 3 .

The systematic review showed ESWT had the best evidence of modalities that have been evaluated. It is typically used for people with non-resolving, persistent symptoms. ${ }^{32} 33$ 37-39 41 Because shock wave therapy is inferior to stretching for acute symptoms, ${ }^{36}$ and based on the clinical reasoning elicitation, ESWT is recommended in the BPG only when patients are failing to recover optimally using the core approach (Figure 3). ESWT had the best evidence of any adjunctive treatments, and has large combined study cohorts, with minimal documented adverse events, demonstrating positive efficacy in the short, medium and long term for most patient reported outcomes. Focussed shock wave is applied so that the peak intensity is deep to the skin and targeted directly at the lesion and had strong positive short term findings on pain of large effect. ${ }^{37}{ }^{38}$ Radial ESWT results in peak intensity at the surface and showed moderate positive effects, again of large effect at all time points for patient reported outcomes. The outcome sizes, when pooled as single arms of multiple studies are high, with this approach taken in order to inform clinicians about the likely actual result of the intervention rather than a comparative effect.

The ESWT results should be viewed considering some limitations that might affect the validity of the results. Many outcomes were poorly reported across trials. Continuous outcomes that evaluated overall pain, first step pain or function were often reported at baseline but not reported at follow up or were presented as the overall success rate with regard to heel pain. For example, Gerdesmeyer ${ }^{32}$ and Gollwitzer ${ }^{37} 39$ presented first step pain, pain while doing activities and pain after application of a dolorimeter at baseline on a continuous scale. However, at follow up they reported scores on a dichotomous scale by defining success as greater than $60 \%$ reduction in heel pain from baseline for at least 2 out of 3 measures that evaluated pain. While it might be advantageous to label participant's outcome as either a 'success' or 'not a success' in response to an intervention, this approach can have a disadvantage. For example, participants that are close to but on opposite sides of the clinical cut off point for success are differently labelled as being categorically different rather than being similar. ${ }^{50}$ Nonetheless, the effect sizes were large, and enough adequately powered trials of high quality were found to guide practice where patients are not improving quickly enough or failing to respond to the core intervention (Figure 3). Further high-quality effectiveness studies are warranted, particularly in people with PHP not responding to the core approach.

Where patients do not respond to core treatment or ESWT then other options are available. Custom orthoses can be considered based on positive evidence of moderate strength and lower effect size than ESWT $^{26}$ for short term outcomes (Figure 3). This is extrapolated from expert interviews and systematic review findings, rather than from trials including failed previous treatment of a specific kind as explicit inclusion criteria.

Off the shelf or custom orthoses are often prescribed for PHP. However, none of the trials included in our review used the same orthosis. All differed in the prescription process, casting technique, shell material, top-covers and modifications thus limiting trial comparison. The prescription of foot orthoses, whether 
customised or prefabricated, commonly involves a process of both education and orthosis modification in an attempt to optimise the dose and biomechanics. ${ }^{51}$ No included RCTS followed this process, possibly limiting effectiveness of custom orthoses. Further, prefabricated orthoses, as used in the included trials, were shown to be ineffective. Therefore, it can be recommended that a single orthosis prescription is not used for all presenting patients, an assertion supported by expert opinion in this study. Given the contrast with custom orthoses, it may be that having a range of prefabricated orthoses may be a suitable strategy as long as prescription is individualised. This approach would be a priority for future cost-effectiveness trials, given the lower cost compared to casting.

Dry needling was found to have a positive effect on pain and function in the short term. Cotchett et al., ${ }^{44}$ revealed a small but significant effect for pain and function based on a statistical approach that included an analysis of covariance. However, our data analysis revealed evidence of no effect, which might have been revealed because comparison of final values included between-participant variability such as differences in baseline pain scores. Based on the latter analysis, dry needling can be considered to have neutral evidence of effect but could be considered as an adjunct intervention to the core approach, with lower priority than orthoses. Trigger point dry needling is also associated with minor adverse events such as needle site pain and to a lesser extent minor bruising. Findings from the interviews indicated that dry needling is not a first line treatment but can be introduced to influence pain and muscle tension when combined with other interventions.

Injection therapy was very carefully assessed, both in the trials and the semi-structured interviews as this is a commonly used intervention. Interestingly, although injection therapy is readily amendable to placebo or sham administration, this has not been performed. It was judged that insertion of a needle with no subsequent drug injection into the plantar fascia could not be considered a no-treatment placebo, as it was likely to have an effect given the findings of Cotchett ${ }^{44}$ with dry needling. Further, injection of any drug or saline would also have chemical and physical effects, which could lead to confounded RCT results. This is an intervention for which placebo control is readily achievable and represents a priority for future research - perhaps in patients where ESWT has failed to yield optimal results.

There was a good match between the qualitative and quantitative results in the study. This was demonstrated with most interventions, where the evidence and expert synthesis was typically coherent. Had there been direct disagreement, then recommendations from quantitative evidence would have prevailed. The agreement was also good where the evidence was unclear, most noticeable in the evidence about exercise-based rehabilitation being almost entirely absent from the main literature and little clarity emerging from the expert interviews. Importantly, patient agreement was very high with the core approach content, indicating it will be acceptable to most people with PHP.

There are many possible uses of this BPG, beyond the immediate utility of guiding clinicians and therapists' practice. BPGs will help to guide patients and clinicians, inform health care commissioners and guide future research. ${ }^{52}$ Patients having access to summary resources such as Figures 2 and 3 should reduce some of the inconsistency they report when seeking guidance from internet and other 
resources. Clinicians can learn from the work, providing resources are available. Commissioners may consider providing the necessary resources - such as ESWT devices. Audit, monitoring and checklist tools do not yet exist, but should be developed and may be a useful stimulus to intervention adoption. Finally, future research may be guided by consideration of the gaps in the evidence base and the expert views on research priorities (Supplementary file 1)

Although we selected only high quality RCTs, the overall quality of the research in the PHP field is low. Of the 362 trials initially assessed for quality using the PEDro and Risk of Bias criteria, only 51 trials met the inclusion criteria. It is argued that summary quality scores used as a criterion for eligibility in metaanalyses might dilute the effect of those items that are closely associated with risk of bias. ${ }^{53}$ As an illustration, the highest quality recent review included 20 trials $^{9}$ which we excluded on quality grounds, and neglected to review 35 studies we included due to the inclusive nature of our search - it being clearly better to restrict search and appraisal returns on quality criteria than others such as type of intervention when generating meaningful practice guides. As such, trials that met the PEDro cut-off score were further evaluated for quality against the standards outlined by the Cochrane Collaboration tool for assessing risk of bias ${ }^{14}$ to ensure there was adequate randomisation, allocation concealment, baseline comparability, blinding of assessors, an intention to treat analysis, and adequate follow up. A number of these variables can have a substantial impact on estimates of treatment effects, ${ }^{15} 5455$ hence our decision to only include those trials with a low risk of bias. The volume and clarity of the available evidence leads to the conclusion that future trials that are not of high quality should not be performed on ethical grounds, while new interventions should have appropriate pre-trial work done on intervention development as per the MRC complex interventions framework. ${ }^{56}$ Effectively, this study represents a strong element of just such intervention development, suitable for further development and testing.

Given the abundance of RCTs that have evaluated interventions for PHP, it is no longer appropriate to include low to moderate quality trials or those with a high risk of bias in systematic reviews and metaanalyses. It could be argued, due to the components of the Pedro score, that this review was biased toward interventions where it is possible to blind the clinician delivering an intervention, and the participant receiving the intervention. However, non-pharmacological interventions (e.g. physical therapy or exercise), where it is not possible to blind the clinician or participant, can still meet other criteria that influence treatment effects such as allocation concealment and blinding of outcome assessors. Researchers are encouraged to apply available methodological strategies to ensure validity of the findings.

Of the 51 included trials, only 9 could be evaluated for primary proof of efficacy and one for secondary proof of efficacy due to the remaining trials being underpowered or not having a suitable control. We considered that a trial with a sample size less than 38 was inadequately powered and was therefore not considered for proof of efficacy in isolation. The pre-specified sample size of 38 was based on the minimal important difference for first step pain measured using a VAS, rather than overall pain, as first step pain is arguably the pathognomonic feature of PHP. The inclusion of trials with small sample sizes 
can inflate effect sizes and these stronger effects might not be representative of the true treatment effect, ${ }^{57}$ which impacts on the validity of the findings and recommendations made to patients, clinicians and funders. It is recommended that researchers perform a priori sample size calculations to ensure the trial has adequate power to detect a difference between groups if a difference truly exists. Researchers are encouraged to calculate the required sample size using the minimal important difference that exists for the VAS and/or FHSQ in this population, ${ }^{17}$ rather than calculating a sample size based on effects from meta-analyses that have included underpowered trials.

This mixed-methods study should be viewed in light of some limitations. First, reviewers who performed the PEDro analysis were not blinded to the study author, which might have introduced bias. Second, the panel of expert clinicians (podiatrists, podiatric surgeons, physiotherapists, sports physician and a rheumatologist) represents the views of the majority but not all disciplines involved in the assessment and management of PHP. Third, the Framework analysis was conducted by a physiotherapist, which could have biased the findings, although this individual has extensive experience in qualitative research and has previously published two reviews ${ }^{22} 58$ that combined best available evidence with expert reasoning. Additionally, the results in this study were extensively debated within the multidisciplinary author team. Finally, there was incomplete reporting in several trials, which limited the available data for meta-analysis.

There are several implications for further research. There is clearly a need for more robust, adequately powered trials with improved reporting of outcomes. At a minimum, researchers must include the mean and a measure of variability at all time points to minimise research waste. There is also a need for an established set of outcome measures that is specific to this population, which will reduce heterogeneity and improve potential for pooling of studies in a meta-analysis. Expert opinion clearly highlighted the importance of patient education as a core treatment for PHP, although no trials included a methodologically robust educational intervention developed in line with best practice such as the MRC framework. ${ }^{56}$ Furthermore, many experts described the value of exercise, particularly strength based programs, although no trial suitable for inclusion in this review attempted to isolate the specific effect of strengthening exercises. Future research should focus on trials that compare one intervention to another of known efficacy. It is not appropriate to compare two or more active interventions that have no proof of efficacy as the superiority of one intervention, over another, might simply reflect a negative effect of an intervention in the comparator group(s). Following new high-quality effectiveness research, the BPG should be revisited and amended.

Views of experts reinforced the need to conduct trials stratified by subgroups such as gender, physical activity level and BMI. However, sub-groups with particularly positive or negative prognosis were not identified in this study, which would be useful to guide research to target those most at risk of poor outcome. Additionally, trials may need to be stratified based on the underlying pathology, with pain experienced in this region of the foot potentially relating to a noxious stimulus in the plantar fascia, muscle, fat, nerve and/or bone. Until the underlying pathology of PHP is appropriately linked to a person's 
signs and symptoms, trials will continue to include participants with a likely heterogeneous group of pathologies subjected to poorly targeted interventions.

Finally, the perceptions and experience of people with PHP identified gaps in how details of the condition and the evidence base is being translated to the patient. Participants highlighted a poor understanding of their condition, including the underlying pathology, causal factors, effectiveness of various treatments, mechanisms behind interventions, knowledge regarding expectations of improvement, and how to progress when treatment is failing. Clinicians must consider how education is being delivered to their patients to ensure there is clear guidance, fears are allayed, and that learning is checked rather than simple information prescription. There is a need for research to explore the educational needs of people with PHP to help guide the development of freely accessible resources to improve self-management of this condition and realisation of optimal effects from the Core intervention.

\section{Conclusions}

\section{What is already known}

- Plantar heel pain is common and can have a negative impact on physical and mental health

- Existing guidelines lack clear, high-quality recommendations for treating people with plantar heel pain

\section{What are the new findings}

- A systematic review and meta-analysis, synthesised with expert clinical reasoning and patient values revealed stretching, foot taping and educational interventions are part of the core approach for people with plantar heel pain.

- A core and stepped approach to the management of people with plantar heel pain was formulated, which will prove immediately useful to clinicians who treat, and to those who suffer from, plantar heel pain.

\section{Declarations}

Acknowledgments: The lead authors (DM and MC) had full access to all the data in the study and take responsibility for the integrity of the data and the accuracy of the data analysis. DM obtained funding, is guarantor, designed the study, selected eligible studies, performed the quality analysis, interviewed experts, constructed the patient survey and analysed and interpreted qualitative data, analysed and interpreted quantitative data, prepared the manuscript. MC conducted the search; selected eligible studies, performed the quality analysis and statistical analysis of quantitative data; interpreted the statistical analysis; prepared the manuscript. CB: designed the study, selected eligible studies, performed the quality analysis; interpreted the statistical analysis of quantitative and qualitative data; reviewed and approved the manuscript. ASBJ: selected eligible studies, performed the quality analysis, interviewed experts, 
reviewed and approved the manuscript. TP: selected eligible studies, performed the quality analysis, reviewed and approved the manuscript. IG: selected eligible studies, performed the quality analysis, reviewed and approved the manuscript. HG: prepared the patient survey and analysed and interpreted qualitative data, reviewed and approved the manuscript MR: selected eligible studies, performed the quality analysis, reviewed and approved the manuscript. BV: performed the quality analysis, reviewed and approved the manuscript.

Acknowledgements go to Dr Marinus Winters who performed an early search of the literature; Dr Saira Chaudhry who advised Dr Said J'Bari during his intercalated degree where some interviews were carried out; Ute Schauberger for an early version of the infographics and Ali Fahmi and Yanzhou Liu for assistance with translation.

Competing Interests: All authors declare no support from any organisation for the submitted work; no financial relationships with any organisations that might have an interest in the submitted work in the previous three years. MC and BV are authors of one of the randomised trials included in this review. MC currently facilitates dry needling courses for podiatrists.

Funding: DM was a recipient of funding from the PPEF for this study, and was part funded by the NIHR/HEE Senior Clinical Lecturer scheme. This article presents independent research part-funded by the National Institute for Health Research (NIHR) CAT SCL-2013-04-003. The views expressed are those of the authors and not necessarily those of the NHS, the NIHR or the Department of Health.

Transparency declaration: The lead authors DM and MC affirms that this manuscript is an honest, accurate, and transparent account of the study being reported; that no important aspects of the study have been omitted; and that any discrepancies from the study as planned and registered have been explained.

Transparency declaration

The lead authors DM and MC affirms that this manuscript is an honest, accurate, and transparent account of the study being reported; that no important aspects of the study have been omitted; and that any discrepancies from the study as planned and registered have been explained.

Ethics declaration

Expert interviews

Verbal informed consent was obtained prior to each interview and ethics approval was granted by the Queen Mary University of London Ethics of Research Committee, UK. 
Patient surveys

Informed consent was obtained electronically by asking the respondents to click on a check box at the beginning of the questionnaire. By clicking on the Yes button and completing the questionnaire, respondents provided consent to their involvement in this project. Ethics approval was granted by the Queen Mary University of London Ethics of Research Committee, UK.

\section{References}

1. Dunn JE, Link CL, Felson DT, et al. Prevalence of foot and ankle conditions in a multiethnic community sample of older adults. Am J Epidemio/2004;159:491-98.

2. Hill CL, Gill TK, Menz HB, et al. Prevalence and correlates of foot pain in a population based study: the North West Adelaide health study. J Foot Ankle Res2008;1

3. Menz HB, Tiedemann A, Kwan MMS, et al. Foot pain in community dwelling older people: an evaluation of the Manchester Foot Pain and Disability Index. Rheumato/2006;45:863-67.

4. Taunton JE, Ryan MB, Clement DB, et al. A retrospective case-control injuries analysis of 2002 running. Br J Sports Med2002;36:95-101. doi: 10.1136/bjsm.36.2.95

5. Martin RL, Davenport TE, Reischl SF, et al. Heel pain-plantar fasciitis: revision 2014. J Orthop Sports Phys Ther2014;44:A1-33. doi: https://dx.doi.org/10.2519/jospt.2014.0303

6. Irving DB, Cook JL, Young MA, et al. Impact of chronic plantar heel pain on health-related quality of life. J Am Podiatr Med Assoc2008;98:283-9.

7. Roberts I, Ker K. How systematic reviews cause research waste. Lancet2015;386:1536.

8. Thomas JL, Christensen JC, Kravitz SR, et al. The diagnosis and treatment of heel pain: a clinical practice guideline-revision 2010. J Foot Ankle Surg2010;49:S1-19. doi: https://dx.doi.org/10.1053/j.jfas.2010.01.001

9. Babatunde OO, Legha A, Littlewood C, et al. Comparative effectiveness of treatment options for plantar heel pain: a systematic review with network meta-analysis. Br J Sports Med2019;53:182-94. doi: 10.1136/bjsports-2017-098998 [published Online First: 2018/06/30]

10. Hoffman T, Bennett S, Del Mar C. Introduction to evidence-based practice. In: Hoffman T, Bennett S, Del Mar C, eds. Evidence-based practice across the health professions. 3rd ed. Chatswood, N.S.W: Churchill Livingstone 2017:3-7.

11. Kamper SJ, Moseley AM, Herbert RD, et al. 15 years of tracking physiotherapy evidence on PEDro, where are we now? Br J Sports Med2015;49:907-09. doi: 10.1136/bjsports-2014-094468

12. Moseley AM, Herbert RD, Maher CG, et al. Reported quality of randomized controlled trials of physiotherapy interventions has improved over time. J Clin Epidemio/2011;64:594-601. 
13. Yamato TP, Maher CG, Koes BW, et al. The PEDro scale had acceptably high convergent validity, construct validity and interrater reliability in evaluating methodological quality of pharmaceutical trials. J Clin Epidemio/2017;86:176-81.

14. Higgins JPT, Green S. Cochrane Handbook for Systematic Reviews of Interventions Version 5.1.0. New Jersey, USA: Wiley Online Library 2011.

15. Wood L, Egger M, Gluud LL, et al. Empirical evidence of bias in treatment effect estimates in controlled trials with different interventions and outcomes: meta-epidemiological study. BMJ2008;336:601-05.

16. Hozo SP, Djulbegovic B, Hozo I. Estimating the mean and variance from the median, range, and the size of a sample. BMC Med Res Methodolo/2005;5:13.

17. Landorf KB, Radford JA, Hudson S. Minimal important difference (MID) of two commonly used outcome measures for foot problems. J Foot Ankle Res2010;14:3:7.

18. van Tulder M, Furlan A, Bombardier C, et al. Updated guidelines for systematic reviews in the Cochrane Collaboration Back Review Group. Spine2003;12:1290-99.

19. Cohen J. Statistical power analysis for the behavioural sciences. 2nd ed. Hillsdale, New Jersey: Lawrence Erlbaum Associates 1988.

20. Rücker G, Cates $C$, Schwarzer G. Methods for including information from multi-arm trials in pairwise meta-analysis. Res Synth Methods2017;8:392-403.

21. Borenstein M, Hedges LV, Higgins JPT, et al. A basic introduction to fixed-effect and random-effects models for meta-analysis. Res Syn Meth2010;1:97-111.

22. Barton CJ, Lack S, Hemmings $S$, et al. The 'Best practice guide to the conservative management of patellofemoral pain: incorporating level 1 evidence with expert clincial reasoning. Br J Sports Med2015;49:923-34.

23. Ritchie J, Lewis J, McNaughton Nicholls C, et al. Qualitative research practice: a guide for social science students and researchers. 2nd ed. Los Angeles: SAGE 2014.

24. Ritchie J, Spencer L. Analysing qualitative data. In: Bryman A, Burgess B, eds. Qualitative data analysis for applied policy research. London: Routledge 1994:173-94.

25. Johnson RB, Onwuegbuzie AJ, Turner LA. Toward a definition of mixed methods research. J Mix Methods Res2007;1:112-33.

26. Landorf KB, Keenan AM, Herbert RD. Effectiveness of foot orthoses to treat plantar fasciitis: a randomized trial. Arch Intern Med2006;166:1305-10. doi:

https://dx.doi.org/10.1001/archinte.166.12.1305

27. Bishop C, Thewlis D, Hillier S. Custom foot orthoses improve first-step pain in individuals with unilateral plantar fasciopathy: a pragmatic randomised controlled trial. BMC Musculoskelet Disord2018;19

28. Oliveira HA, Jones A, Moreira E, et al. Effectiveness of total contact insoles in patients with plantar fasciitis. J Rheumato/2015;42:870-8. doi: https://dx.doi.org/10.3899/jrheum.140429 
29. Wrobel JS, Fleischer AE, Crews RT, et al. A randomized controlled trial of custom foot orthoses for the treatment of plantar heel pain. J Am Podiatr Med Assoc2015;105:281-94. doi: https://dx.doi.org/10.7547/13-122.1

30. Baldassin V, Gomes CR, Beraldo PS. Effectiveness of prefabricated and customized foot orthoses made from low-cost foam for noncomplicated plantar fasciitis: a randomized controlled trial. Arch Phys Med Rehabi/2009;90:701-6. doi: https://dx.doi.org/10.1016/j.apmr.2008.11.002

31. Winemiller MH, Billow RG, Laskowski ER, et al. Effect of magnetic vs sham-magnetic insoles on plantar heel pain: a randomized controlled trial. JAMA2003;290:1474-78. doi: http://dx.doi.org/10.1001/jama.290.11.1474

32. Gerdesmeyer L, Frey C, Vester $\mathrm{J}$, et al. Radial extracorporeal shock wave therapy is safe and effective in the treatment of chronic recalcitrant plantar fasciitis: results of a confirmatory randomized placebo-controlled multicenter study. Am J Sports Med2008;36:2100-9. doi: https://dx.doi.org/10.1177/0363546508324176

33. Ibrahim MI, Donatelli RA, Hellman M, et al. Long-term results of radial extracorporeal shock wave treatment for chronic plantar fasciopathy: A prospective, randomized, placebo-controlled trial with two years follow-up. J Orthop Res2017;35:1532-38. doi: 10.1002/jor.23403

34. Hocaoglu S, Vurdem UE, Cebicci MA, et al. Comparative effectiveness of radial extracorporeal shockwave therapy and ultrasound-guided local corticosteroid injection treatment for plantar fasciitis. J Am Podiatr Med Assoc2017;107:192-99. doi: https://dx.doi.org/10.7547/14-114

35. Lohrer $\mathrm{H}$, Nauck T, Dorn-Lange NV, et al. Comparison of radial versus focused extracorporeal shock waves in plantar fasciitis using functional measures. Foot Ankle Int2010;31:1-9. doi: https://dx.doi.org/10.3113/FAl.2010.0001

36. Rompe JD, Cacchio A, Weil L, Jr., et al. Plantar fascia-specific stretching versus radial shock-wave therapy as initial treatment of plantar fasciopathy. J Bone Joint Surg Am2010;92:2514-22. doi: https://dx.doi.org/10.2106/JBJS.I.01651

37. Gollwitzer H, Saxena A, DiDomenico LA, et al. Clinically relevant effectiveness of focused extracorporeal shock wave therapy in the treatment of chronic plantar fasciitis: a randomized, controlled multicenter study. J Bone Joint Surg Am2015;97:701-8. doi: https://dx.doi.org/10.2106/JBJS.M.01331

38. Speed CA, Nichols D, Wies J, et al. Extracorporeal shock wave therapy for plantar fasciitis. A double blind randomised controlled trial. J Orthop Res2003;21:937-40. doi: https://dx.doi.org/10.1016/S0736-0266(03)00048-2

39. Gollwitzer H, Diehl P, von Korff A, et al. Extracorporeal shock wave therapy for chronic painful heel syndrome: a prospective, double blind, randomized trial assessing the efficacy of a new electromagnetic shock wave device. J Foot Ankle Surg2007;46:348-57. doi: https://dx.doi.org/10.1053/j.jfas.2007.05.011

40. Buchbinder R, Ptasznik R, Gordon J, et al. Ultrasound-guided extracorporeal shock wave therapy for plantar fasciitis: a randomized controlled trial. JAMA2002;288:1364-72. 
41. Rompe JD, Decking J, Schoellner C, et al. Shock wave application for chronic plantar fasciitis in running athletes. A prospective, randomized, placebo-controlled trial. Am J Sports Med2003;31:26875. doi: https://dx.doi.org/10.1177/03635465030310021901

42. Kudo P, Dainty K, Clarfield M, et al. Randomized, placebo-controlled, double-blind clinical trial evaluating the treatment of plantar fasciitis with an extracoporeal shockwave therapy (ESWT) device: a North American confirmatory study. J Orthop Res2006;24:115-23. doi: https://dx.doi.org/10.1002/jor.20008

43. Rompe JD, Meurer A, Nafe B, et al. Repetitive low-energy shock wave application without local anesthesia is more efficient than repetitive low-energy shock wave application with local anesthesia in the treatment of chronic plantar fasciitis. J Orthop Res2005;23:931-41. doi: https://dx.doi.org/10.1016/j.orthres.2004.09.003

44. Cotchett MP, Munteanu SE, Landorf KB. Effectiveness of trigger point dry needling for plantar heel pain: a randomized controlled trial. Phys Ther2014;94:1083-94. doi: https://dx.doi.org/10.2522/ptj.20130255

45. Young MA, Cook JL, Webster KE. The effect of topical wheatgrass cream on chronic plantar fasciitis: a randomized, double-blind, placebo-controlled trial. Complement Ther Med2006;14:3-9. doi: https://dx.doi.org/10.1016/j.ctim.2005.07.003

46. Radford JA, Landorf KB, Buchbinder R, et al. Effectiveness of calf muscle stretching for the shortterm treatment of plantar heel pain: a randomised trial. BMC Musculoskelet Disord2007;8:36. doi: https://dx.doi.org/10.1186/1471-2474-8-36

47. Radford JA, Landorf KB, Buchbinder R, et al. Effectiveness of low-Dye taping for the short-term treatment of plantar heel pain: a randomised trial. BMC Musculoskelet Disord2006;7:64. doi: https://dx.doi.org/10.1186/1471-2474-7-64

48. Brouwers MC, Kerkvliet K, Spithoff K, et al. The Agree Reporting Checklist: a tool to improve reporting of clinical practice guidelines. BMJ2016;6:i4852.

49. Lin I, Wiles LK, Waller R, et al. Poor overall quality of clinical practice guidelines for musculoskeletal pain: a systematic review. Br J Sports Med2018;52:337-43. doi: 10.1136/bjsports-2017-098375

50. Altman AR, Royston P. The cost of dichotomising continuous variables. BMJ2006;332:1080.

51. Kirby KA, Spooner SK, Scherer PR, et al. Foot orthoses. Foot Ankle Spec2012;5:334-43. doi: 10.1177/1938640012458900 [published Online First: 2012/09/12]

52. Morrissey D. Guidelines and pathways for clinical practice in tendinopathy: their role and development. J Orthop Sports Phys Ther2015;45:819-22.

53. Armijo-Olivo S, da Costa BR, Cummings G, et al. PEDro or Cochrane to assess the quality of clinical trials? A meta-epidemiological study. PLoS One2015; e0132634

54. Schulz KF, Chalmers I, Hayes RJ, et al. Empirical evidence of bias: dimensions of methodological quality associated with estimates of treatment effects in controlled trials. JAMA 1995;273:408-12.

55. Pildal J, Hrobjartsson A, Jørgensen KJ, et al. Impact of allocation concealment on conclusions drawn from meta-analyses of randomized trials. Internat J Epidemio/2007;36:847-57. 
56. Craig P, Dieppe P, Macintyre S, et al. Developing and evaluating complex interventions: the new Medical Research Council guidance. BMJ2008;337:a1655. doi: 10.1136/bmj.a1655

57. Dechartres A, Trinquart L, Boutron I, et al. Influence of trial sample size on treatment effect estimates: meta-epidemiological study. BMJ2013;346

58. Rowe V, Hemmings S, Barton CJ, et al. Conservative management of midportion achilles tendinopathy. Sports Med2012;42:941-67.

\section{Figures}

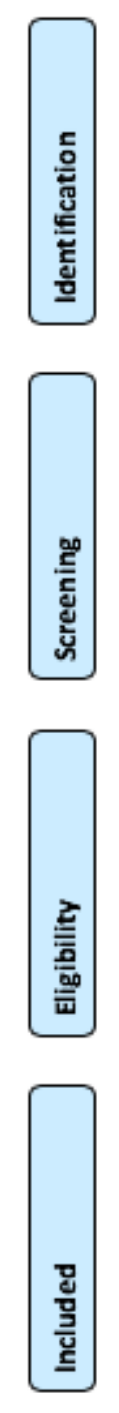

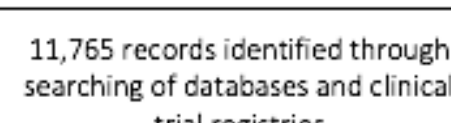
trial registries

\section{Figure 1}

Flow of information through the review 


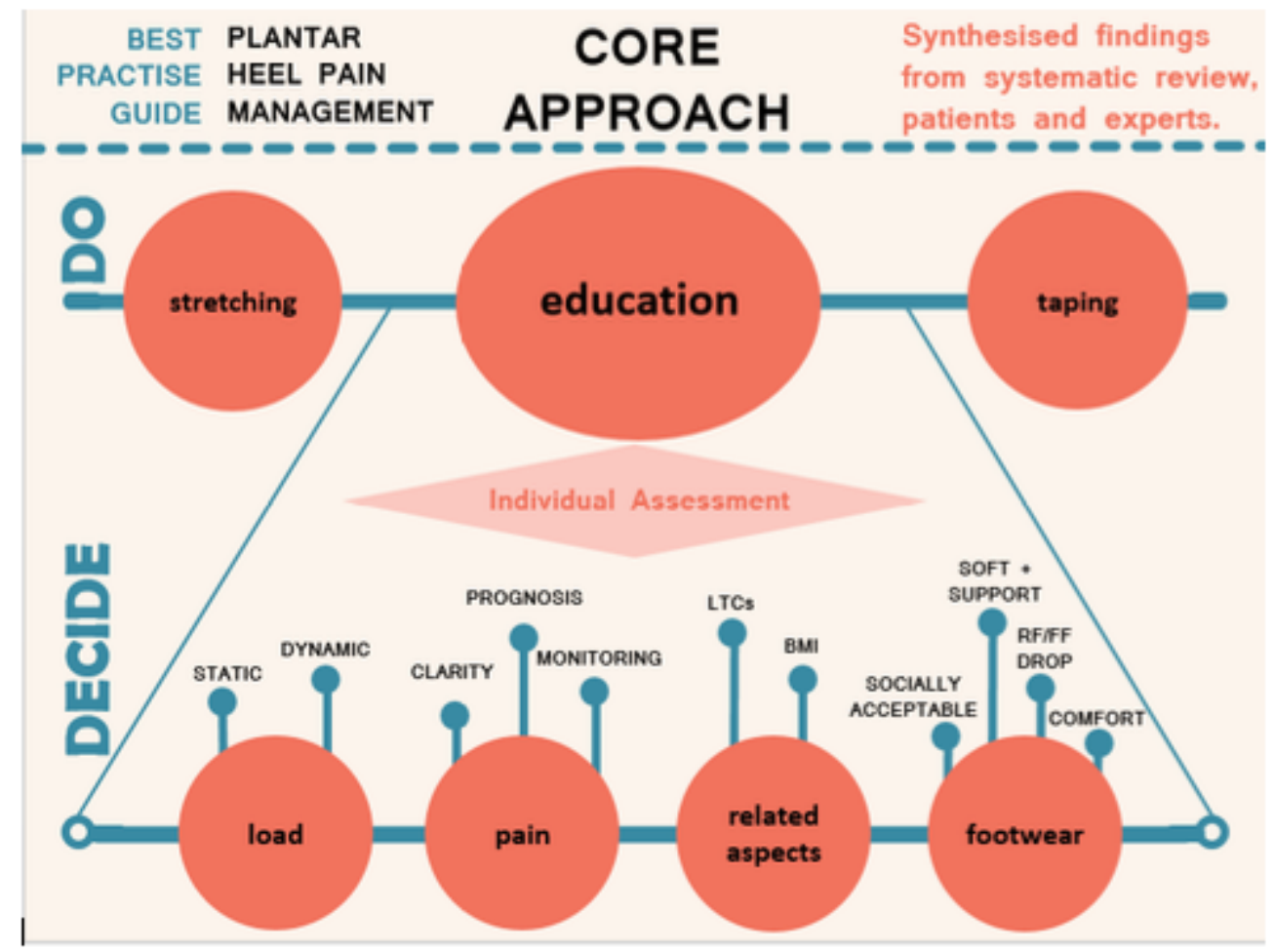

Figure 2: Core approach to the management of plantar heel pain based on the best available evidence, expert opinion and the patient voice. The top layer ('DO') of taping, stretching and education are required initial interventions with each patient. The individual assessment ('DECIDE') is of which specific educational aspects are needed. LTCS = long-term conditions. RF = rearfoot, FF = forefoot, $\mathrm{BMI}=$ body mass index.

\section{Figure 2}

Core approach to the management of plantar heel pain based on the best available evidence, expert opinion and the patient voice. The top layer ('DO') of taping, stretching and education are required initial interventions with each patient. The individual assessment ('DECIDE') is of which specific educational aspects are needed. LTCs = long-term conditions. RF = rearfoot, FF = forefoot, BMI = body mass index. 


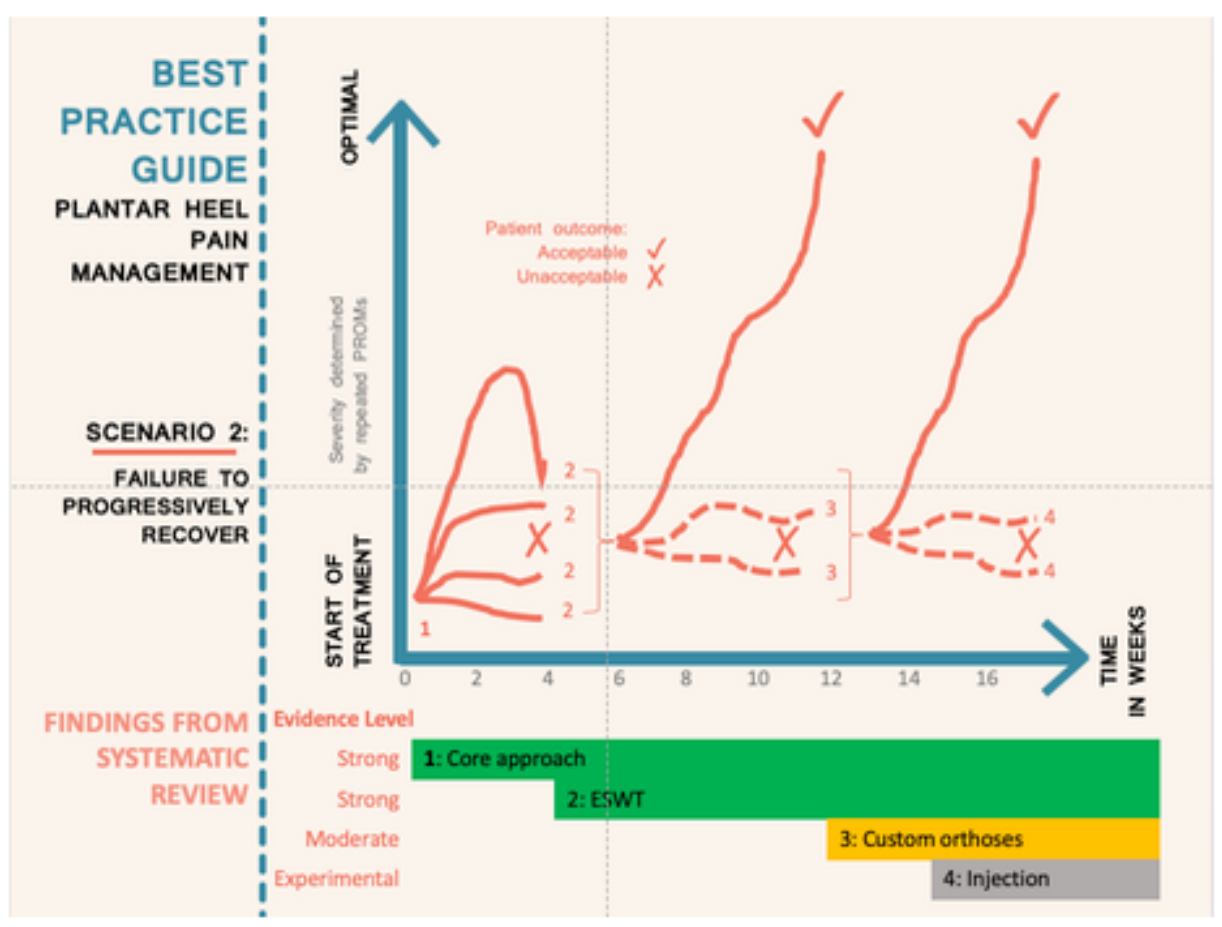

Figure 3: Management approach for plantar heel pain when a person progressively fails to recover with addition of ESWT at 4 weeks if the core approach is not working and then addition of orthoses at 12 weeks if there is still sub-optimal improvement.

\section{Figure 3}

Management approach for plantar heel pain when a person progressively fails to recover with addition of ESWT at 4 weeks if the core approach is not working and then addition of orthoses at 12 weeks if there is still sub-optimal improvement.

\section{Supplementary Files}

This is a list of supplementary files associated with this preprint. Click to download.

- OnlineonlysupplementsforResearchSquare.docx 